\title{
Linear Yang-Mills Theory as a Homotopy AQFT
}

\author{
Marco Benini $^{1,2}{ }_{\mathbb{D}}$, Simen Bruinsma ${ }^{3}$ (D) Alexander Schenkel $^{3}(\mathbb{D}$ \\ 1 Fachbereich Mathematik, Universität Hamburg, Bundesstr. 55, 20146 Hamburg, Germany. \\ E-mail: marco.benini@uni-hamburg.de; benini@dima.unige.it \\ 2 Dipartimento di Matematica, Università di Genova, Via Dodecaneso 35, 16146 Genoa, Italy \\ 3 School of Mathematical Sciences, University of Nottingham, University Park, Nottingham NG7 2RD, UK. \\ E-mail: simen.bruinsma@nottingham.ac.uk; alexander.schenkel@nottingham.ac.uk
}

\begin{abstract}
It is observed that the shifted Poisson structure (antibracket) on the solution complex of Klein-Gordon and linear Yang-Mills theory on globally hyperbolic Lorentzian manifolds admits retarded/advanced trivializations (analogs of retarded/ advanced Green's operators). Quantization of the associated unshifted Poisson structure determines a unique (up to equivalence) homotopy algebraic quantum field theory (AQFT), i.e. a functor that assigns differential graded $*$-algebras of observables and fulfills homotopical analogs of the AQFT axioms. For Klein-Gordon theory the construction is equivalent to the standard one, while for linear Yang-Mills it is richer and reproduces the BRST/BV field content (gauge fields, ghosts and antifields).
\end{abstract}

\section{Contents}

1. Introduction and Summary . . . . . . . . . . . . . . . . . 185

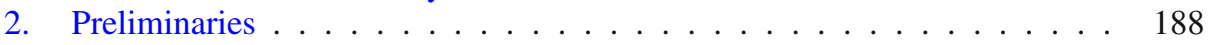

2.1 Green's operators . . . . . . . . . . . . . . . . . . . . . . . 188

2.2 Chain complexes . . . . . . . . . . . . . . . . . . . . . . . 190

3. Field and Solution Complexes . . . . . . . . . . . . . . . . . . . . . . 192

4. Shifted and Unshifted Poisson Structures . . . . . . . . . . . . . . . . 196

4.1 Klein-Gordon theory . . . . . . . . . . . . . . . . . . . . 200

4.2 Linear Yang-Mills theory . . . . . . . . . . . . . . . . . . . 201

5. Quantization ........................ 204

6. Functoriality and Homotopy AQFT Axioms _ . . . . . . . . . . 206

A. Technical Details for Proposition $5.3 \ldots \ldots \ldots$

\section{Introduction and Summary}

Because of their outstanding significance in physics and their intricate connection to mathematics, quantum gauge theories continuously attract a high level of attention 
throughout different fields of research. In the context of algebraic quantum field theory (AQFT) [HK64,BFV03], which is a powerful axiomatic framework for quantum field theory on Lorentzian manifolds, it is a long-standing open problem to identify the characteristic features of quantum gauge theories and their gauge symmetries from a modelindependent perspective. To support these more abstract developments, concrete examples of quantum gauge theories were constructed in the context of AQFT. Most of these studies focused on the case of Yang-Mills theory with structure group $\mathbb{R}$ or $U(1)$, see e.g. [SDH14, BDS14, BDHS14,FL16, Ben16, BSS16], but there also exist similar developments for e.g. linearized gravity [FH13, BDM14, Kha16, Kha18] and linearized supergravity [HS13]. In addition to such non-interacting models, examples of perturbatively interacting quantum gauge theories were constructed in [Hol08,FR12,FR13, TZ18] by means of an appropriate adaption of the BRST/BV formalism to AQFT.

One of the main conceptual insights of these studies was the observation that quantum gauge theories, when formulated traditionally in terms of gauge-invariant on-shell observable algebras, are in conflict with crucial axioms of AQFT. The first observation [DL12] was that quantum gauge theories may violate the isotony axiom of AQFT, which demands that the push-forward $\mathfrak{A}(f): \mathfrak{A}(M) \rightarrow \mathfrak{A}(N)$ of observables along every spacetime embedding $f: M \rightarrow N$ is an injective map. It was later understood that the violation of isotony is due to topological charges in quantum gauge theories, e.g. electric and magnetic fluxes in Abelian Yang-Mills theory, and hence it is a feature that is expected on physical grounds, see e.g. [SDH14, BDS14, BDHS14, Ben16, BSS16, BBSS17] for a detailed explanation. The second observation is more subtle as it is related to local-to-global properties (i.e. descent) of AQFTs. Within the traditional formulation in terms of gauge-invariant on-shell observable algebras, quantum gauge theories have very poor local-to-global properties as witnessed for example by the observation in [DL12,FL16] that Fredenhagen's universal algebra (which is a certain local-to-global construction) for Abelian Yang-Mills theory fails to encode crucial gauge theoretic features such as Dirac's charge quantization and Aharonov-Bohm phases. It was later understood and emphasized in [BSS15] that the failure of (too naive versions of) local-to-global constructions is due to higher categorical structures in classical and quantum gauge theories, which are neglected (i.e. truncated) when working in a traditional AQFT setting that is based on gauge-invariant on-shell observables.

Our approach towards resolving this conflict at the interface of AQFT and gauge theory is the recent homotopical AQFT program [BSS15, BS17, BSS18, BSW17, BSW19a, BSW19b,BS18], whose aim is to refine the foundations of AQFT by introducing new concepts from higher category theory. We refer to [BS19] for a recent summary and stateof-the-art review of this approach. Informally speaking, the main difference between a homotopy AQFT and an ordinary AQFT is that it assigns to each spacetime a higher categorical algebra in contrast to an ordinary $*$-algebra of observables, such that suitable homotopy coherent analogs of the AQFT axioms hold true. These statements can be made precise by using techniques from operad theory [BSW17,BSW19a,BSW19b]. Such higher observable algebras should be understood as quantizations of function algebras on higher categorical spaces called (derived) stacks, which are crucial for the description of field and solution spaces in a gauge theory, see e.g. [Sch13] and [BS19] for an introduction and also [BSS18] for a concrete description of the Yang-Mills stack. In the context of linear and perturbative quantum gauge theory, the higher field and solution spaces may be described by chain complexes of vector spaces and the higher quantum observable algebras by differential graded $*$-algebras. A more physical approach to such higher cat- 
egorical structures is given by the BRST/BV formalism, which has already found many interesting applications in perturbative AQFT, see e.g. [Hol08,FR12,FR13, TZ18].

One of the most pressing current issues of the homotopical AQFT program is that there is up to now no fully worked out physical example of a quantum gauge theory in this framework. (Various oversimplified toy-models appeared previously in e.g. [BS17, BSW19b, BS18].) It is the aim of the present paper to address this issue by constructing a first proper example of a homotopy AQFT, namely linear quantum Yang-Mills theory with structure group $\mathbb{R}$ on globally hyperbolic Lorentzian manifolds. Let us emphasize that, even though linear Yang-Mills theory is clearly one of the simplest examples of a gauge theory, its construction as a homotopy AQFT is far from trivial because one has to work consistently within a higher categorical context.

A central role in our construction is played by (a linear analog of) the derived critical locus of the linear Yang-Mills action functional, which yields a chain complex that encodes very refined information about the solutions to the linear Yang-Mills equation. By general results of derived algebraic geometry [PTVV13, CPTVV17, Pri18], this chain complex carries a canonical shifted Poisson structure, which is the crucial ingredient in the factorization algebra approach to quantum field theory by Costello and Gwilliam [CG17]. One of our main observations in this paper is that this shifted Poisson structure is trivial in homology due to the geometry of globally hyperbolic Lorentzian manifolds and that it can be trivialized by two different kinds of homotopies that play a similar role as retarded/advanced Green's operators in ordinary field theory. Taking the difference between a retarded and an advanced trivialization allows us to define an unshifted Poisson structure and hence to study the canonical quantization of linear Yang-Mills theory. One of the technical challenges that we address in this paper is a homotopical analysis of the construction sketched above, which is required to ensure that it is meaningful within our higher categorical context, i.e. compatible with quasi-isomorphisms of chain complexes and chain homotopies between Poisson structures. For this we shall use techniques from both model category theory [Hov99,Hir03] and homotopical category theory [DHKS04, Rie14]. In order to make the bulk of this paper accessible to a broader audience, we limit our use of such homotopical techniques to the bare minimum that is required to ensure consistency of our results.

Let us now explain in more detail our constructions and results by outlining the content of the present paper: In Sect. 2 we recall some preliminary results concerning retarded/advanced Green's operators for Green hyperbolic operators on globally hyperbolic Lorentzian manifolds and concerning chain complexes of vector spaces. These techniques will be frequently used throughout the whole paper. In Sect. 3 we introduce a flexible concept of field complexes for linear gauge theories and compute the solution complexes corresponding to a quadratic action functional via a linear analog of the derived critical locus construction. We apply these techniques to two explicit examples, given by Klein-Gordon and linear Yang-Mills theory on globally hyperbolic Lorentzian manifolds, and explain how they relate to the BRST/BV formalism from physics. In particular, the derived critical locus construction produces the field content of the BRST/BV formalism, i.e. fields, ghosts and antifields, together with the relevant differentials.

In Sect. 4 we describe and analyze the shifted Poisson structure on the solution complex that exists canonically due to its construction as a derived critical locus. (In the terminology of the BRST/BV formalism, this is called the antibracket.) Our main novel observation is that, for Klein-Gordon and linear Yang-Mills theory on globally hyperbolic Lorentzian manifolds, this shifted Poisson structure is trivial in homology and that it can be trivialized by two distinct types of homotopies that play a similar role to 
retarded/advanced Green's operators in ordinary field theory. We formalize this insight by introducing an abstract concept of retarded/advanced trivializations (Definition 4.4). We prove that these trivializations exist for our two running examples and that they are unique in an appropriate sense: For Klein-Gordon theory they are unique, while for linear Yang-Mills theory they are not unique in a strict sense but rather unique up to chain homotopies, which is an appropriate and expected relaxation within our higher categorical context of the uniqueness result for retarded/advanced Green's operators in ordinary field theory. Taking the difference between (a compatible pair of) a retarded and an advanced trivialization allows us to define an unshifted Poisson structure (Definition 4.8), which is again unique up to chain homotopies.

In Sect. 5 we study in detail homotopical properties of the canonical commutation relations (CCR) quantization of unshifted Poisson complexes into differential graded *-algebras. Our main result in this section is Proposition 5.3, which proves that CCR quantization is compatible with weak equivalences of Poisson complexes and also with homotopies of unshifted Poisson structures. This proof requires a rather technical result that is proven in "Appendix A". Hence, the examples obtained by our construction in Sect. 4 can be quantized consistently. We spell out the quantization of Klein-Gordon and linear Yang-Mills theory in this approach explicitly in Examples 5.5 and 5.6.

In Sect. 6 we investigate functoriality of our constructions and answer affirmatively our initial question whether they define examples of homotopy AQFTs (Definition 6.1). A key ingredient for these studies is an appropriate concept of natural retarded/advanced trivializations (Definition 6.5) and of natural unshifted Poisson structures (Definition 6.8). Our construction in Sect. 4 determines such natural structures for both Klein-Gordon and linear Yang-Mills theory, see Proposition 6.7. The main result of this paper is Theorem 6.19, which proves that our construction yields a description of Klein-Gordon and linear Yang-Mills theory as homotopy AQFTs. Concerning uniqueness (up to natural weak equivalences) of our construction via natural retarded/advanced trivializations, we observe that there are subtle differences between Klein-Gordon and linear Yang-Mills theory. While our construction determines Klein-Gordon theory uniquely (up to natural weak equivalences) on the category Loc of all globally hyperbolic Lorentzian manifolds, we can currently only ensure uniqueness (up to natural weak equivalences) for linear Yang-Mills theory on each slice category Loc $/ \bar{M}$, for $\bar{M} \in \mathbf{L o c}$. (See Theorem 6.19 and Remarks 6.20 and 6.21 for the details.) In AQFT terminology, this means that, even though we successfully provide a construction of linear Yang-Mills theory as a homotopy AQFT in the locally covariant framework [BFV03], we can currently only ensure that each of its restrictions to a Haag-Kastler style homotopy AQFT on a fixed spacetime $\bar{M} \in \mathbf{L o c}$ is determined uniquely (up to natural weak equivalences) by our methods. This potential non-uniqueness of linear quantum Yang-Mills theory in the locally covariant setting is linked to features of the category of globally hyperbolic spacetimes Loc which, in contrast to the slice categories Loc $\bar{M}$, has no terminal object.

\section{Preliminaries}

2.1. Green's operators. We briefly review those aspects of the theory of Green hyperbolic operators on globally hyperbolic Lorentzian manifolds that are required for this work. The reader is referred to [BGP07, Bar15] for the details.

Let $M$ be an oriented and time-oriented globally hyperbolic Lorentzian manifold of dimension $m \geq 2$. Let $F \rightarrow M$ be a finite-rank real vector bundle and denote its vector space of sections by $\mathfrak{F}(M)=\Gamma^{\infty}(M, F)$. A linear differential operator $P$ : 
$\mathfrak{F}(M) \rightarrow \mathfrak{F}(M)$ is called Green hyperbolic if it admits retarded and advanced Green's operators $G^{ \pm}: \mathfrak{F}_{\mathrm{c}}(M) \rightarrow \mathfrak{F}(M)$, where the subscript c denotes compactly supported sections. Recall that a retarded/advanced Green's operator is by definition a linear map $G^{ \pm}: \mathfrak{F}_{\mathrm{c}}(M) \rightarrow \mathfrak{F}(M)$ which satisfies the following properties:

(i) $G^{ \pm} P \varphi=\varphi$, for all $\varphi \in \mathfrak{F}_{\mathrm{c}}(M)$;

(ii) $P G^{ \pm} \varphi=\varphi$, for all $\varphi \in \mathfrak{F}_{\mathrm{c}}(M)$;

(iii) $\operatorname{supp}\left(G^{ \pm} \varphi\right) \subseteq J_{M}^{ \pm}(\operatorname{supp}(\varphi))$, for all $\varphi \in \mathfrak{F}_{\mathrm{c}}(M)$, where $J_{M}^{ \pm}(S) \subseteq M$ denotes the causal future/past of a subset $S \subseteq M$.

It was proven in [Bar15] that retarded/advanced Green's operators are necessarily unique and that they admit unique extensions

$$
G^{ \pm}: \mathfrak{F}_{\mathrm{pc} / \mathrm{fc}}(M) \longrightarrow \mathfrak{F}_{\mathrm{pc} / \mathrm{fc}}(M)
$$

to sections with past/future compact support, such that the three properties above hold true for all $\varphi \in \mathfrak{F}_{\mathrm{pc} / \mathrm{fc}}(M)$. (Recall that $s \in \mathfrak{F}(M)$ has past/future compact support if there exists a Cauchy surface $\Sigma \subset M$ such that $\operatorname{supp}(s) \subseteq J_{M}^{ \pm}(\Sigma)$.) The difference $G:=G^{+}-G^{-}: \mathfrak{F}_{\mathrm{c}}(M) \rightarrow \mathfrak{F}(M)$ of the retarded and advanced Green's operator (on compactly supported sections) is often called the causal propagator. From the properties of $G^{ \pm}$it follows that

$$
0 \longrightarrow \mathfrak{F}_{\mathrm{c}}(M) \stackrel{P}{\longrightarrow} \mathfrak{F}_{\mathrm{c}}(M) \stackrel{G}{\longrightarrow} \mathfrak{F}_{\mathrm{sc}}(M) \stackrel{P}{\longrightarrow} \mathfrak{F}_{\mathrm{sc}}(M) \longrightarrow 0
$$

is an exact sequence, where the subscript sc denotes sections of spacelike compact support. (Recall that $s \in \mathfrak{F}(M)$ has spacelike compact support if there exists a compact subset $K \subseteq M$ such that $\operatorname{supp}(s) \subseteq J_{M}^{+}(K) \cup J_{M}^{-}(K)$.) In particular, this implies that $P G=0=G P$.

For every vector bundle $F \rightarrow M$ that is endowed with a fiber metric $h$ one can define the integration pairing

$$
\left\langle s, s^{\prime}\right\rangle:=\int_{M} h\left(s, s^{\prime}\right) \operatorname{vol}_{M},
$$

for all $s, s^{\prime} \in \mathfrak{F}(M)$ with compactly overlapping support. Let us consider two such vector bundles $F_{1} \rightarrow M$ and $F_{2} \rightarrow M$ with fiber metrics and a linear differential operator $Q: \mathfrak{F}_{1}(M) \rightarrow \mathfrak{F}_{2}(M)$. There exists a formal adjoint differential operator $Q^{*}: \mathfrak{F}_{2}(M) \rightarrow \mathfrak{F}_{1}(M)$ defined by

$$
\left\langle s_{2}, Q s_{1}\right\rangle_{2}=\left\langle Q^{*} s_{2}, s_{1}\right\rangle_{1},
$$

for all $s_{1} \in \mathfrak{F}_{1}(M)$ and $s_{2} \in \mathfrak{F}_{2}(M)$ with compactly overlapping support. A linear differential operator $P: \mathfrak{F}(M) \rightarrow \mathfrak{F}(M)$ with source and target determined by the same vector bundle $F \rightarrow M$ with fiber metric $h$ is called formally self-adjoint if $P^{*}=P$. If $P: \mathfrak{F}(M) \rightarrow \mathfrak{F}(M)$ is a formally self-adjoint Green hyperbolic operator, then its Green's operators satisfy

$$
\left\langle\varphi, G^{+} \varphi^{\prime}\right\rangle=\left\langle G^{-} \varphi, \varphi^{\prime}\right\rangle,
$$

for all $\varphi, \varphi^{\prime} \in \mathfrak{F}_{\mathrm{c}}(M)$. This implies that the causal propagator $G=G^{+}-G^{-}$is formally skew-adjoint, i.e.

$$
\left\langle\varphi, G \varphi^{\prime}\right\rangle=-\left\langle G \varphi, \varphi^{\prime}\right\rangle
$$

for all $\varphi, \varphi^{\prime} \in \mathfrak{F}_{\mathrm{c}}(M)$. 
Example 2.1. The following class of examples is most relevant for this work. Consider the $p$-th exterior power $F=\bigwedge^{p} T^{*} M \rightarrow M$ of the cotangent bundle. Then $\mathfrak{F}(M)=$ $\Omega^{p}(M)$ is the vector space of $p$-forms. The orientation and Lorentzian metric on $M$ define a Hodge operator $*: \Omega^{p}(M) \rightarrow \Omega^{m-p}(M)$ and thereby a fiber metric, whose integration pairing reads as

$$
\langle\omega, \zeta\rangle=\int_{M} \omega \wedge * \zeta,
$$

for all $\omega, \zeta \in \Omega^{p}(M)$ with compactly overlapping support. The de Rham differential $\mathrm{d}: \Omega^{p}(M) \rightarrow \Omega^{p+1}(M)$ is a linear differential operator and its formal adjoint is the codifferential $\delta:=\mathrm{d}^{*}: \Omega^{p+1}(M) \rightarrow \Omega^{p}(M)$. The d'Alembert operator on $p$-forms is defined by

$$
\square:=\delta \mathrm{d}+\mathrm{d} \delta: \Omega^{p}(M) \longrightarrow \Omega^{p}(M)
$$

and it is formally self-adjoint. Because of $\mathrm{d}^{2}=0$ and $\delta^{2}=0$, the d'Alembert operators in different degrees are related by

$$
\mathrm{d} \square=\square \mathrm{d}, \quad \delta \square=\square \delta .
$$

The d'Alembert operators are Green hyperbolic and because of (2.9) the retarded/advanced Green's operators in different degrees are related by

$$
\mathrm{d} G^{ \pm}=G^{ \pm} \mathrm{d}, \quad \delta G^{ \pm}=G^{ \pm} \delta .
$$

Finally, we note that the Klein-Gordon-type operators $\square-m^{2}: \Omega^{p}(M) \rightarrow \Omega^{p}(M)$, where $m \in \mathbb{R}_{\geq 0}$ is a mass term, are formally self-adjoint Green hyperbolic operators too.

2.2. Chain complexes. Chain complexes play a crucial role in formulating and proving our results in this paper. The present subsection contains a brief review of basic aspects of the theory of chain complexes that are necessary for this work. This will in particular allow us to fix the notations and conventions that we employ in the main part of this paper. For more details on chain complexes we refer to [Wei94] and also to [Hov99].

Let us fix a field $\mathbb{K}$ of characteristic zero and consider $\mathbb{K}$-vector spaces. In the main sections $\mathbb{K}$ will be either the real numbers $\mathbb{R}$ or the complex numbers $\mathbb{C}$. A chain complex is a family of vector spaces $\left\{V_{n}\right\}_{n \in \mathbb{Z}}$ together with a differential, i.e. a family of linear maps $\left\{\mathrm{d}_{n}: V_{n} \rightarrow V_{n-1}\right\}_{n \in \mathbb{Z}}$ such that $\mathrm{d}_{n-1} \mathrm{~d}_{n}=0$ for all $n \in \mathbb{Z}$. To simplify notations, we often denote this data collectively by $V$ and write $\mathrm{d}: V_{n} \rightarrow V_{n-1}$ for every component of the differential. A chain map $f: V \rightarrow W$ is a family of linear maps $\left\{f_{n}: V_{n} \rightarrow\right.$ $\left.W_{n}\right\}_{n \in \mathbb{Z}}$ that is compatible with the differentials, i.e. $\mathrm{d} f_{n}=f_{n-1} \mathrm{~d}$ for all $n \in \mathbb{Z}$. We denote by $\mathbf{C h}_{\mathbb{K}}$ the category of chain complexes of $\mathbb{K}$-vector spaces with chain maps as morphisms.

The tensor product $V \otimes W \in \mathbf{C h}_{\mathbb{K}}$ of two chain complexes $V, W \in \mathbf{C h}_{\mathbb{K}}$ is defined by

$$
(V \otimes W)_{n}:=\bigoplus_{m \in \mathbb{Z}} V_{m} \otimes W_{n-m},
$$


for all $n \in \mathbb{Z}$, together with the differential obtained by the graded Leibniz rule $\mathrm{d}(v \otimes$ $w):=\mathrm{d} v \otimes w+(-1)^{m} v \otimes \mathrm{d} w$, for all $v \in V_{m}$ and $w \in W_{n-m}$. Note that the $\otimes$ on the right-hand side of (2.11) is the tensor product of vector spaces. The unit for this tensor product is given by $\mathbb{K} \in \mathbf{C h}_{\mathbb{K}}$, which we regard as a chain complex concentrated in degree 0 with trivial differential. The tensor product of chain complexes is symmetric via the chain isomorphisms $\gamma: V \otimes W \rightarrow W \otimes V$ defined by the usual sign-rule $\gamma(v \otimes w):=(-1)^{m k} w \otimes v$, for all $v \in V_{m}$ and $w \in W_{k}$. Finally, the mapping complex $\underline{\operatorname{hom}}(V, W) \in \mathbf{C h}_{\mathbb{K}}$ between two chain complexes $V, W \in \mathbf{C h}_{\mathbb{K}}$ is defined by

$$
\underline{\operatorname{hom}}(V, W)_{n}:=\prod_{m \in \mathbb{Z}} \operatorname{Lin}\left(V_{m}, W_{n+m}\right),
$$

for all $n \in \mathbb{Z}$, where Lin denotes the vector space of linear maps between vector spaces, together with the "adjoint" differential $\partial: \underline{\operatorname{hom}}(V, W)_{n} \rightarrow \underline{\operatorname{hom}}(V, W)_{n-1}$ defined by

$$
\partial L:=\left\{\mathrm{d} L_{m}-(-1)^{n} L_{m-1} \mathrm{~d}: V_{m} \rightarrow W_{n-1+m}\right\}_{m \in \mathbb{Z}} \in \underline{\operatorname{hom}}(V, W)_{n-1},
$$

for all $L=\left\{L_{m}: V_{m} \rightarrow W_{n+m}\right\}_{m \in \mathbb{Z}} \in \underline{\operatorname{hom}}(V, W)_{n}$. In summary, this endows $\mathbf{C h}_{\mathbb{K}}$ with the structure of a closed symmetric monoidal category.

To every chain complex $V \in \mathbf{C h}_{\mathbb{K}}$ one can assign its homology $H_{\bullet}(V)=$ $\left\{H_{n}(V)\right\}_{n \in \mathbb{Z}}$, which is the graded vector space defined by $H_{n}(V):=\operatorname{Ker}\left(\mathrm{d}: V_{n} \rightarrow\right.$ $\left.V_{n-1}\right) / \operatorname{Im}\left(\mathrm{d}: V_{n+1} \rightarrow V_{n}\right)$, for all $n \in \mathbb{Z}$. A chain map $f: V \rightarrow W$ is called a quasiisomorphism if it induces an isomorphism $H_{\bullet}(f): H_{\bullet}(V) \rightarrow H_{\bullet}(W)$ in homology. Quasi-isomorphic chain complexes should be regarded as "being the same", which can be made precise by using techniques from model category theory [Hov99] or $\infty$-category theory [LurHTT,LurHA]. It is proven in [Hov99] that $\mathbf{C h}_{\mathbb{K}}$ carries the structure of a symmetric monoidal model category, whose weak equivalences are the quasi-isomorphisms and fibrations are the degree-wise surjective maps. Every object in the model category $\mathbf{C h}_{\mathbb{K}}$ is both fibrant and cofibrant. Readers who are not familiar with model categories should read the previous statements informally as that "there exists technology to perform a variety of constructions with chain complexes that are compatible with quasiisomorphisms". In this paper we try to keep the model categorical technicalities to a bare minimum. We refer to [BS19] for a detailed explanation why such techniques are conceptually crucial for formalizing (quantum) gauge theories.

Let us also briefly recall the concept of chain homotopies. A chain homotopy between two chain maps $f, g: V \rightarrow W$ is a family of linear maps $\lambda=\left\{\lambda_{n}: V_{n} \rightarrow W_{n+1}\right\}_{n \in \mathbb{Z}}$ such that $f_{n}-g_{n}=\mathrm{d} \lambda_{n}+\lambda_{n-1} \mathrm{~d}$, for all $n \in \mathbb{Z}$. This definition can be rephrased very conveniently by using the mapping complexes from (2.12). Note that a chain map $f$ : $V \rightarrow W$ is precisely a 0 -cycle in $\underline{\operatorname{hom}}(V, W) \in \mathbf{C h}_{\mathbb{K}}$, i.e. an element $f \in \underline{\operatorname{hom}}(V, W)_{0}$ of degree 0 satisfying $\partial f=0$. A chain homotopy between two chain maps $f, g: V \rightarrow W$ is precisely a 1-chain in $\underline{\operatorname{hom}}(V, W) \in \mathbf{C h}_{\mathbb{K}}$, i.e. an element $\lambda \in \underline{\operatorname{hom}}(V, W)_{1}$ of degree 1 , such that $\partial \lambda=f-g$. Observe that such chain homotopies exist if and only if the homology class $[f-g] \in H_{0}(\underline{\operatorname{hom}}(V, W))$ vanishes. This picture immediately generalizes to higher homotopies: Given two chain homotopies $\lambda, \lambda^{\prime} \in \underline{\operatorname{hom}}(V, W)_{1}$ between $f, g: V \rightarrow W$, then $\lambda-\lambda^{\prime}$ is a 1-cycle in $\underline{\operatorname{hom}}(V, W) \in \mathbf{C h}_{\mathbb{K}}$, i.e. $\partial\left(\lambda-\lambda^{\prime}\right)=0$. A (higher) chain homotopy between $\lambda$ and $\lambda^{\prime}$ is a 2-chain $\chi \in \underline{\operatorname{hom}}(V, W)_{2}$ such that $\partial \chi=\lambda-\lambda^{\prime}$. Observe that such (higher) chain homotopies exist if and only if the homology class $\left[\lambda-\lambda^{\prime}\right] \in H_{1}(\underline{\operatorname{hom}}(V, W))$ vanishes. The pattern for even higher chain homotopies is now evident.

We conclude this subsection by fixing our conventions for shiftings (also called suspensions) of chain complexes. Given any $V \in \mathbf{C h}_{\mathbb{K}}$ and $p \in \mathbb{Z}$, we define $V[p] \in \mathbf{C h}_{\mathbb{K}}$ 
by $V[p]_{n}:=V_{n-p}$, for all $n \in \mathbb{Z}$, together with the differential $\mathrm{d}_{n}^{V[p]}:=(-1)^{p} \mathrm{~d}_{n-p}^{V}$, where we temporarily used a superscript on $\mathrm{d}$ in order to indicate the relevant chain complex. Note that $V[p][q]=V[p+q]$, for all $p, q \in \mathbb{Z}$, and that $V[0]=V$. From the definition of the tensor product (2.11), one finds that $V[p] \cong \mathbb{K}[p] \otimes V$. For every $V, W \in \mathbf{C h}_{\mathbb{K}}$ and $p \in \mathbb{Z}$, there exists a chain isomorphism hom $(V, W[p]) \cong \operatorname{hom}(V, W)[p]$ determined by the components

$$
\begin{aligned}
\underline{\operatorname{hom}}(V, W[p])_{n} & \longrightarrow \underline{\operatorname{hom}(V, W)[p]_{n},} \\
\left\{L_{m}: V_{m} \rightarrow W[p]_{n+m}\right\}_{m \in \mathbb{Z}} & \longmapsto\left\{L_{m}: V_{m} \rightarrow W_{n-p+m}\right\}_{m \in \mathbb{Z}} .
\end{aligned}
$$

\section{Field and Solution Complexes}

Let $M$ be an oriented and time-oriented globally hyperbolic Lorentzian manifold of dimension $m \geq 2$. In this section all chain complexes will be over $\mathbb{R}$, i.e. the relevant category is $\mathbf{C h}_{\mathbb{R}}$. Our aim is to investigate the solution chain complexes for a class of linear gauge field theories on $M$, which we will obtain from a derived critical locus construction. The following definition will be self-explanatory after Examples 3.2 and 3.3.

Definition 3.1. A field complex on $M$ is a chain complex

$$
\mathfrak{F}(M):=\left(\mathfrak{F}_{0}(M) \stackrel{Q}{\longleftarrow} \mathfrak{F}_{1}(M)\right)
$$

concentrated in homological degrees 0 and 1 , where

(i) $\mathfrak{F}_{n}(M)=\Gamma^{\infty}\left(M, F_{n}\right)$ is the vector space of sections of a finite-rank real vector bundle $F_{n} \rightarrow M$ with fiber metric $h_{n}$, for $n=0,1$, and

(ii) $Q: \mathfrak{F}_{1}(M) \rightarrow \mathfrak{F}_{0}(M)$ is a linear differential operator.

Example 3.2. Scalar fields on $M$ are described by the field complex

$$
\left(\Omega^{0}(M) \stackrel{0}{\longleftarrow} 0\right)
$$

concentrated in homological degree 0 . The fiber metrics are the ones obtained from the Hodge operator, see Example 2.1. The elements in degree 0 are interpreted as scalar fields $\Phi \in \Omega^{0}(M)$ and triviality of the complex in degree 1 means that there are no gauge transformations, as it should be in a scalar field theory.

Example 3.3. Gauge fields with structure group $G=\mathbb{R}$ on $M$ are described by the field complex

$$
\left(\Omega^{1}(M) \stackrel{\mathrm{d}}{\longleftarrow} \Omega^{0}(M)\right)
$$

where $\mathrm{d}$ is the de Rham differential. The fiber metrics are the ones obtained from the Hodge operator, see Example 2.1. The elements in degree 0 are interpreted as gauge fields $A \in \Omega^{1}(M)$ and the elements in degree 1 as gauge transformations $\epsilon \in \Omega^{0}(M)$. The differential d encodes how gauge transformations act on gauge fields, i.e. $A \rightarrow A+\mathrm{d} \epsilon$.

Remark 3.4. We would like to mention very briefly that Definition 3.1 admits an obvious generalization to longer complexes 


$$
\mathfrak{F}(M)=\left(\mathfrak{F}_{0}(M) \stackrel{Q_{1}}{\longleftarrow} \mathfrak{F}_{1}(M) \stackrel{Q_{2}}{\longleftarrow} \mathfrak{F}_{2}(M) \stackrel{Q_{3}}{\longleftarrow} \cdots\right),
$$

where each $\mathfrak{F}_{n}(M)=\Gamma^{\infty}\left(M, F_{n}\right)$ is the vector space of sections of a finite-rank real vector bundle $F_{n} \rightarrow M$ with fiber metric $h_{n}$ and each $Q_{n}: \mathfrak{F}_{n}(M) \rightarrow \mathfrak{F}_{n-1}(M)$ is a linear differential operator. Such generalization is relevant for the description of higher gauge theories, which include gauge transformations between gauge transformations. For example, the complex

$$
\left(\Omega^{p}(M) \stackrel{\mathrm{d}}{\longleftarrow} \Omega^{p-1}(M) \overleftarrow{\mathrm{d}} \cdots \stackrel{\mathrm{d}}{\longleftarrow} \Omega^{0}(M)\right)
$$

describes $p$-form gauge fields $A \in \Omega^{p}(M)$ with gauge transformations $A \rightarrow A+\mathrm{d} \Lambda$, for $\Lambda \in \Omega^{p-1}(M)$, 2-gauge transformations $\Lambda \rightarrow \Lambda+\mathrm{d} \lambda$, for $\lambda \in \Omega^{p-2}(M)$, and so on. Our results and constructions in this paper apply to this more general case as well, however we decided to focus on 1-gauge theories as in Definition 3.1 in order to improve readability. In particular, our main examples of interest are described by 2-term field complexes, see Examples 3.2 and 3.3.

In order to encode the dynamics, we consider a formally self-adjoint linear differential operator

$$
P: \mathfrak{F}_{0}(M) \longrightarrow \mathfrak{F}_{0}(M),
$$

which we interpret as the equation of motion operator for the fields of the theory. The corresponding quadratic action functional

$$
S\left(s_{0}\right):=\frac{1}{2}\left\langle s_{0}, P s_{0}\right\rangle=\frac{1}{2} \int_{M} h_{0}\left(s_{0}, P s_{0}\right) \operatorname{vol}_{M}
$$

is given by the integration pairing (2.3). This action is gauge-invariant if and only if $P$ satisfies

$$
P Q=0,
$$

which from now on is always assumed. Because $P$ is formally self-adjoint, it follows that

$$
0=(P Q)^{*}=Q^{*} P^{*}=Q^{*} P \text {. }
$$

The variation of the action defines a section $\delta^{\mathrm{v}} S: \mathfrak{F}(M) \rightarrow T^{*} \mathfrak{F}(M)$ of the cotangent bundle over $\mathfrak{F}(M)$. As in [BS19, Section 3.4], we define the latter as the product complex

$$
T^{*} \mathfrak{F}(M):=\mathfrak{F}(M) \times \mathfrak{F}_{\mathrm{c}}(M)^{*}
$$

with

$$
\mathfrak{F}_{\mathrm{c}}(M)^{*}:=\left(\mathfrak{F}_{1}^{(-1)}(M) \stackrel{-Q^{*}}{\longleftarrow} \mathfrak{F}_{0}(M)\right)
$$

the smooth dual of the compactly supported field complex $\mathfrak{F}_{\mathrm{c}}(M)$. Here and in the following we use round brackets to indicate homological degrees. Explicitly, we obtain

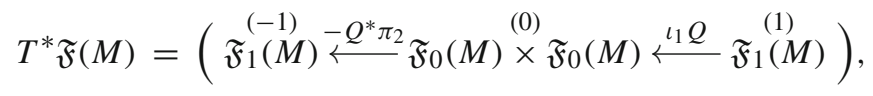


where $\iota_{1}: \mathfrak{F}_{0}(M) \rightarrow \mathfrak{F}_{0}(M) \oplus \mathfrak{F}_{0}(M)=\mathfrak{F}_{0}(M) \times \mathfrak{F}_{0}(M)$ denotes the inclusion into the first factor and $\pi_{2}: \mathfrak{F}_{0}(M) \times \mathfrak{F}_{0}(M) \rightarrow \mathfrak{F}_{0}(M)$ the projection onto the second factor. The chain map $\delta^{\mathrm{v}} S: \mathfrak{F}(M) \rightarrow T^{*} \mathfrak{F}(M)$ obtained by varying the action then reads explicitly as

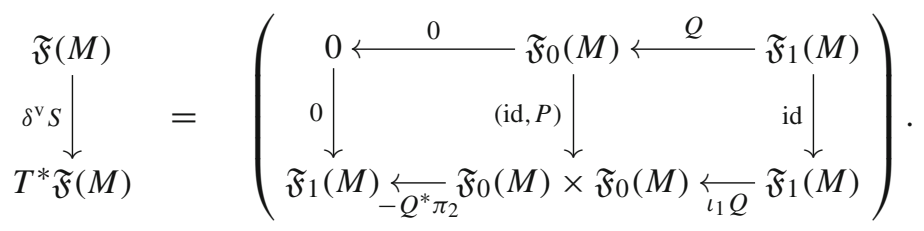

Note the appearance of the equation of motion operator $P: \mathfrak{F}_{0}(M) \rightarrow \mathfrak{F}_{0}(M)$ in the middle vertical arrow. Hence, in order to enforce the equation of motion, we have to intersect $\delta^{\mathrm{v}} S$ with the zero-section

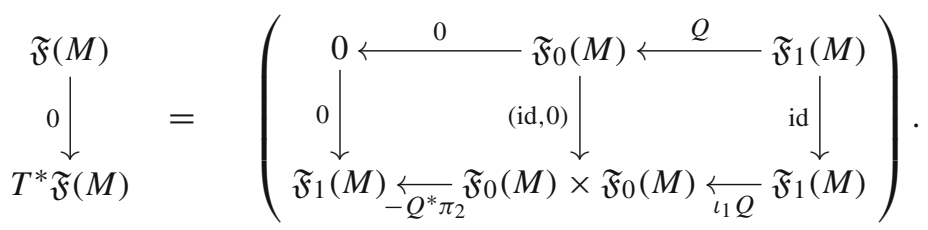

This is the content of the following.

Definition 3.5. Let $\mathfrak{F}(M)$ be a field complex on $M$ and $P: \mathfrak{F}_{0}(M) \rightarrow \mathfrak{F}_{0}(M)$ a formally self-adjoint linear differential operator satisfying (3.8). The corresponding solution complex on $M$ is defined as the derived critical locus of the action functional $S$ in (3.7). Concretely, it is given by the homotopy pullback

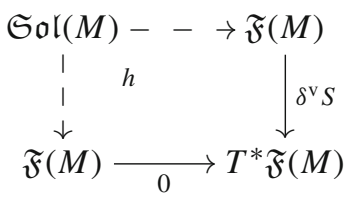

in the model category $\mathbf{C h}_{\mathbb{R}}$.

Remark 3.6. We would like to add an informal discussion of the important role of homotopy pullbacks (see e.g. [Hov99,Hir03]) for the benefit of those readers who are not familiar with model categories. First, let us note that if (3.12) would be an ordinary categorical pullback, then it would enforce the equation of motion in a strict fashion, i.e. $P s_{0}=0$. There are however problems with this naive approach, because it is not guaranteed that replacing $\mathfrak{F}(M)$ by a quasi-isomorphic chain complex will yield quasiisomorphic solution complexes $\mathfrak{S o l}(M)$. (Recall that quasi-isomorphic chain complexes should be regarded as "being the same".) A homotopy pullback is a suitable deformation (called a derived functor) of the ordinary pullback that does not suffer from this problem. Consequently, our chain complex $\mathfrak{S o l}(M)$ from Definition 3.5 is invariant (up to quasi-isomorphisms) under changing $\mathfrak{F}(M)$ by quasi-isomorphisms. One should think of our solution complex $\operatorname{Sol}(M)$ as enforcing the equation of motion $P s_{0}=0$ in only a weak sense, i.e. "up to homotopy". 
Proposition 3.7. A model for the solution complex $\mathfrak{S o l}(M)$ from Definition 3.5 is given by

$$
\operatorname{Sol}(M)=\left(\mathfrak{F}_{1}(M) \stackrel{(-2)}{\stackrel{Q^{*}}{\longleftarrow}} \mathfrak{F}_{0}^{(-1)}(M) \stackrel{P}{\longleftarrow} \mathfrak{F}_{0}(M) \stackrel{(0)}{\longleftarrow} \stackrel{(1)}{\longleftarrow} \mathfrak{F}_{1}(M)\right) .
$$

Proof. The homotopy pullback in (3.12) can be computed by using some basic model category technology, yielding the result in (3.13). The proof for linear Yang-Mills theory in [BS19, Proposition 3.21] generalizes in a straightforward way to our present scenario and hence it will not be repeated.

Example 3.8. For the scalar field complex from Example 3.2, we choose the massive Klein-Gordon operator $P=\square-m^{2}: \Omega^{0}(M) \rightarrow \Omega^{0}(M)$. The action in (3.7) is then the usual Klein-Gordon action

$$
S(\Phi)=\frac{1}{2}\left\langle\Phi, \square \Phi-m^{2} \Phi\right\rangle=\frac{1}{2} \int_{M}\left(\mathrm{~d} \Phi \wedge * \mathrm{~d} \Phi-m^{2} \Phi^{2} \operatorname{vol}_{M}\right) .
$$

The corresponding solution complex from Proposition 3.7 explicitly reads as

$$
\mathfrak{S o l}^{\mathrm{KG}}(M)=\left(0 \stackrel{0}{\longleftarrow} \Omega^{0}(M) \stackrel{\square-m^{2}}{\longleftarrow} \Omega^{0}(M) \longleftarrow 0 \quad 0\right) .
$$

The components of this complex admit a physical interpretation in terms of the BRST/BV formalism:

- the fields in degree 0 are the scalar fields $\Phi \in \Omega^{0}(M)$;

- the fields in degree -1 are the antifields $\Phi^{\ddagger} \in \Omega^{0}(M)$.

Note that only the zeroth homology of $\mathfrak{S o l}^{\mathrm{KG}}(M)$ is non-vanishing. It is given by the ordinary solution space $H_{0}\left(\mathfrak{S o l}^{\mathrm{KG}}(M)\right)=\left\{\Phi \in \Omega^{0}(M): \square \Phi-m^{2} \Phi=0\right\}$ of KleinGordon theory. It follows that $\mathfrak{S o l}^{\mathrm{KG}}(M)$ is quasi-isomorphic to its zeroth homology $H_{0}\left(\mathfrak{S o l}^{\mathrm{KG}}(M)\right)$, regarded as a chain complex concentrated in degree 0 . In other words, for Klein-Gordon theory on $M$ it does not make any difference if we work with the solution complex $\mathfrak{S}_{0} \mathfrak{l}^{\mathrm{KG}}(M)$ or with the ordinary solution space $H_{0}\left(\mathfrak{S o l}^{\mathrm{KG}}(M)\right)$.

Example 3.9. For the gauge field complex from Example 3.3, we choose the linear YangMills operator $P=\delta \mathrm{d}: \Omega^{1}(M) \rightarrow \Omega^{1}(M)$. The action in (3.7) is then the usual linear Yang-Mills action

$$
S(A)=\frac{1}{2}\langle A, \delta \mathrm{d} A\rangle=\frac{1}{2}\langle\mathrm{~d} A, \mathrm{~d} A\rangle=\frac{1}{2} \int_{M} F \wedge * F,
$$

with $F=\mathrm{d} A \in \Omega^{2}(M)$ the field strength. The corresponding solution complex from Proposition 3.7 explicitly reads as

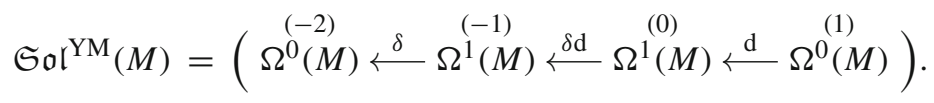

The components of this complex admit a physical interpretation in terms of the BRST/BV formalism:

- the fields in degree 0 are the gauge fields $A \in \Omega^{1}(M)$;

- the fields in degree 1 are the ghost fields $c \in \Omega^{0}(M)$; 
- the fields in degrees -1 and -2 are the antifields $A^{\ddagger} \in \Omega^{1}(M)$ and $c^{\ddagger} \in \Omega^{0}(M)$.

The homologies of $\mathfrak{S o r}^{\mathrm{YM}}(M)$ can be computed explicitly and admit a physical interpretation.

- $H_{1}\left(\mathfrak{S o r}^{\mathrm{YM}}(M)\right) \cong H_{\mathrm{dR}}^{0}(M)$ is the zeroth de Rham cohomology of $M$. It describes those gauge transformations that act trivially on gauge fields, i.e. it encodes the extent to which the gauge group fails to act freely. This homology is never trivial, because the dimension of the vector space $H_{\mathrm{dR}}^{0}(M) \cong \mathbb{R}^{\pi_{0}(M)}$ is given by the number of connected components of the manifold $M$.

- $H_{0}\left(\mathfrak{S}_{\mathfrak{o r}} \mathrm{Y}^{\mathrm{M}}(M)\right)=\left\{A \in \Omega^{1}(M): \delta \mathrm{d} A=0\right\} / \mathrm{d} \Omega^{0}(M)$ is the usual vector space of gauge equivalence classes of linear Yang-Mills solutions.

- $H_{-1}\left(\mathfrak{S o l}^{\mathrm{YM}}(M)\right) \cong H_{\delta}^{1}(M) \cong H_{\mathrm{dR}}^{m-1}(M)$ is the first $\delta$-cohomology or equivalently the $m-1$-th de Rham cohomology of $M$. It captures obstructions to solving the inhomogeneous linear Yang-Mills equation $\delta \mathrm{d} A=j$ with $j \in \Omega_{\delta}^{1}(M)$ a $\delta$-closed 1-form, i.e. $\delta j=0$. For the explicit computation of $H_{-1}\left(\mathfrak{S o l}^{\mathrm{YM}}(M)\right)$ one uses standard techniques from the theory of normally hyperbolic operators [BGP07,Bar15] in order to prove that $\delta \mathrm{d} A=j$ admits a solution $A$ if and only if $j=\delta \zeta$ is $\delta$-exact.

- $H_{-2}\left(\operatorname{Sol}^{\mathrm{YM}}(M)\right) \cong H_{\delta}^{0}(M) \cong H_{\mathrm{dR}}^{m}(M) \cong 0$ is the zeroth $\delta$-cohomology or equivalently the $m$-th de Rham cohomology of $M$. This is trivial because every globally hyperbolic Lorentzian manifold is diffeomorphic to a product manifold $M \cong \mathbb{R} \times \Sigma$.

We in particular observe that $\mathfrak{S}_{\mathfrak{o}} \mathfrak{l}^{\mathrm{YM}}(M)$ can not be quasi-isomorphic to a chain complex concentrated in degree 0 , hence it contains more refined information than the vector space of gauge equivalence classes of linear Yang-Mills solutions, i.e. the zeroth homology $H_{0}\left(\mathfrak{S o l}^{\mathrm{YM}}(M)\right)$. It is the latter that is traditionally considered in the AQFT literature, see e.g. [SDH14,BDS14,BDHS14,FL16,Ben16,BSS16].

\section{Shifted and Unshifted Poisson Structures}

A general result of derived algebraic geometry [PTVV13,CPTVV17, Pri18] states that every derived critical locus comes endowed with a shifted symplectic structure and hence a shifted Poisson structure. Such shifted Poisson structures play a fundamental role in the factorization algebra approach to quantum field theory by Costello and Gwilliam [CG17]. We explain below that the solution complex $\operatorname{Sol}(M)$ from Proposition 3.7 carries a natural shifted Poisson structure. For our two examples given by Klein-Gordon and linear Yang-Mills theory, we shall make the interesting observation that this shifted Poisson structure defines a trivial homology class, which crucially relies on our hypothesis that $M$ is a globally hyperbolic Lorentzian manifold. In these examples there exist two distinct types of chain homotopies (called retarded and advanced) that trivialize the shifted Poisson structure, which play an analogous role to the retarded and advanced Green's operators in ordinary field theory, see e.g. [BGP07,BDH13]. Taking the difference between a compatible pair of retarded and advanced trivializations allows us to define an unshifted Poisson structure on $\operatorname{Sol}(M)$, which is the necessary ingredient for canonical commutation relations (CCR) quantization in Sect. 5.

Both the shifted and unshifted Poisson structures will be defined on the smooth dual of the solution complex from Proposition 3.7, which should be interpreted as a chain complex of linear observables. 
Definition 4.1. The complex of linear observables for the solution complex $\operatorname{Sol}(M)$ from (3.13) is defined by

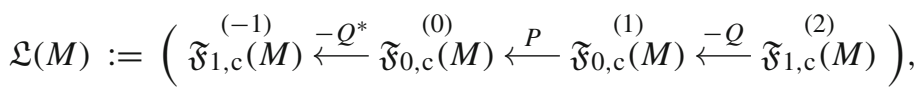

where the subscript $\mathrm{c}$ denotes compactly supported sections. The integration pairings (2.3) define evaluation chain maps

$$
\begin{aligned}
& \langle\cdot, \cdot\rangle: \mathfrak{L}(M) \otimes \mathfrak{S o l}(M) \longrightarrow \mathbb{R} \\
& \langle\cdot, \cdot\rangle: \operatorname{Sol}(M) \otimes \mathfrak{L}(M) \longrightarrow \mathbb{R}
\end{aligned}
$$

between linear observables and solutions.

In order to define the shifted Poisson structure on $\operatorname{Sol}(M)$, let us consider the [1]shifting (see Sect. 2.2) of the solution complex $\mathfrak{S o l}(M)$ in (3.13), i.e.

$$
\operatorname{Sol}(M)[1]=\left(\mathfrak{F}_{1}^{(M)} \stackrel{(-1)}{\longleftarrow} Q^{*} \mathfrak{F}_{0}\left(\stackrel{(0)}{\longleftarrow} \stackrel{-P}{\longleftarrow} \mathfrak{F}_{0}^{(1)}(M) \stackrel{-Q}{\longleftarrow} \mathfrak{F}_{1}^{(2)}(M)\right),\right.
$$

and observe that the inclusion maps $\iota: \mathfrak{F}_{n, \mathrm{c}}(M) \rightarrow \mathfrak{F}_{n}(M)$ of compactly supported sections define a chain map

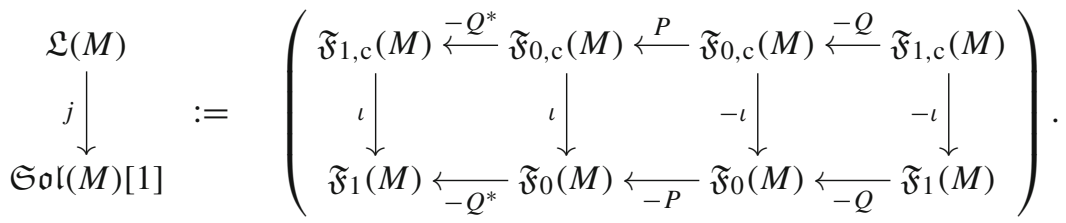

Definition 4.2. The shifted Poisson structure on the solution complex $\operatorname{Sol}(M)$ in (3.13) is the chain map $\Upsilon: \mathfrak{L}(M) \otimes \mathfrak{L}(M) \rightarrow \mathbb{R}[1]$ defined by the composition

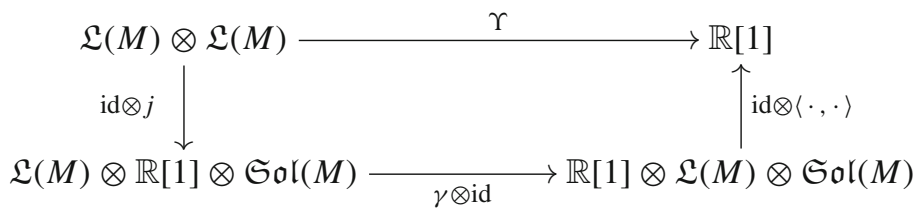

where $\gamma$ is the symmetric braiding in $\mathbf{C h}_{\mathbb{R}}, \mathfrak{L}(M)$ is the complex of linear observables (4.1) for $\mathfrak{S o l}(M)$ and we implicitly used the isomorphism $\operatorname{Sol}(M)[1] \cong \mathbb{R}[1] \otimes \mathfrak{S o l}(M)$, see Sect. 2.2.

Remark 4.3. In the terminology of the BRST/BV formalism, the shifted Poisson bracket is called the antibracket.

As we explain in detail in the two subsections below, our examples given by KleinGordon and linear Yang-Mills theory on an oriented and time-oriented globally hyperbolic Lorentzian manifold $M$ have the interesting feature that the homology class $[j]=0 \in H_{0}(\underline{\operatorname{hom}}(\mathfrak{L}(M), \operatorname{Sol}(M)[1]))$ of the chain map (4.4) is trivial and as a consequence the homology class $[\Upsilon]=0 \in H_{0}(\underline{\operatorname{hom}}(\mathfrak{L}(M) \otimes \mathfrak{L}(M), \mathbb{R}[1]))$ of the shifted Poisson structure is trivial too. We shall obtain an interpretation of the trivializations of $j$ as analogs of the Green's operators in ordinary field theory. Before working out the 
details for our two examples, we would like to introduce some general terminology and definitions that will be useful for this task. First, let us introduce the past/future compact analog of the complex (4.1), i.e.

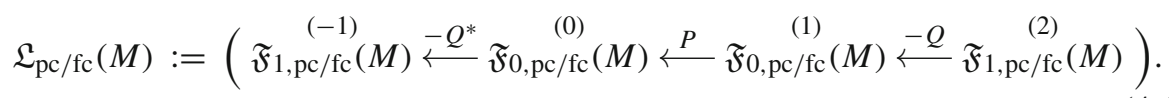

Observe that the chain map $j$ in (4.4) factors through the canonical inclusions $\iota$ : $\mathfrak{L}(M) \rightarrow \mathfrak{L}_{\mathrm{pc} / \mathrm{fc}}(M)$, i.e. we have a commutative triangle

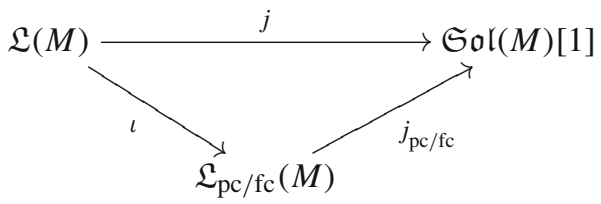

where $j_{\mathrm{pc} / \mathrm{fc}}$ is the evident extension of the chain map (4.4) to sections with past/future compact support.

Definition 4.4. A retarded/advanced trivialization is a contracting homotopy of the chain complex $\mathfrak{L}_{\mathrm{pc} / \mathrm{fc}}(M)$, i.e. a 1-chain $\Lambda^{ \pm} \in \underline{\operatorname{hom}}\left(\mathfrak{L}_{\mathrm{pc} / \mathrm{fc}}(M), \mathfrak{L}_{\mathrm{pc} / \mathrm{fc}}(M)\right)_{1}$ such that id $=\partial \Lambda^{ \pm}$.

The following are some simple properties of retarded/advanced trivializations.

Lemma 4.5. (a) If $\Lambda^{ \pm} \in \underline{\operatorname{hom}}\left(\mathfrak{L}_{\mathrm{pc} / \mathrm{fc}}(M), \mathfrak{L}_{\mathrm{pc} / \mathrm{fc}}(M)\right)_{1}$ is a retarded/advanced trivialization, then $j=\partial\left(j_{\mathrm{pc} / \mathrm{fc}} \Lambda^{ \pm} \iota\right)$ and $\Upsilon=\partial\left((\mathrm{id} \otimes\langle\cdot, \cdot\rangle)(\gamma \otimes \mathrm{id})\left(\mathrm{id} \otimes\left(j_{\mathrm{pc} / \mathrm{fc}} \Lambda^{ \pm} \iota\right)\right)\right)$. In particular, the homology classes $[j]=0$ and $[\Upsilon]=0$ are trivial.

(b) If $\Lambda^{ \pm}, \widetilde{\Lambda}^{ \pm} \in \underline{\operatorname{hom}}\left(\mathfrak{L}_{\mathrm{pc} / \mathrm{fc}}(M), \mathfrak{L}_{\mathrm{pc} / \mathrm{fc}}(M)\right)_{1}$ are two retarded/advanced trivializations, then $\tilde{\Lambda}^{ \pm}-\Lambda^{ \pm}=\partial \lambda^{ \pm}$for some 2 -chain $\lambda^{ \pm} \in \underline{\operatorname{hom}}\left(\mathfrak{L}_{\mathrm{pc} / \mathrm{fc}}(M), \mathfrak{L}_{\mathrm{pc} / \mathrm{fc}}(M)\right)_{2}$.

(c) If $\Lambda^{ \pm} \in \underline{\operatorname{hom}}\left(\mathfrak{L}_{\mathrm{pc} / \mathrm{fc}}(M), \mathfrak{L}_{\mathrm{pc} / \mathrm{fc}}(M)\right)_{1}$ is a pair of retarded/advanced trivializations, then

$$
\Lambda:=j_{\mathrm{pc}} \Lambda^{+} \iota-j_{\mathrm{fc}} \Lambda^{-} \iota \in \underline{\operatorname{hom}}(\mathfrak{L}(M), \operatorname{Sol}(M)[1])_{1}
$$

is a 1-cycle, i.e. $\partial \Lambda=0$. Via the chain isomorphism (2.13), this defines a chain map $\Lambda: \mathfrak{L}(M) \rightarrow \operatorname{Sol}(M)$ to the unshifted solution complex.

Proof. Items (a) and (c) are straightforward checks. For item (b) we note that the homology of $\mathfrak{L}_{\mathrm{pc} / \mathrm{fc}}(M)$ is trivial because $\Lambda^{ \pm}$is by definition a contracting homotopy of $\mathfrak{L}_{\mathrm{pc} / \mathrm{fc}}(M)$. Because all objects in $\mathbf{C h}_{\mathbb{R}}$ are fibrant and cofibrant, the mapping complex functor hom preserves quasi-isomorphisms, hence the homology of $\underline{\operatorname{hom}}\left(\mathfrak{L}_{\mathrm{pc} / \mathrm{fc}}(M), \mathfrak{L}_{\mathrm{pc} / \mathrm{fc}}\left(\overline{M))}\right.\right.$ is trivial too. Since $\partial\left(\Lambda^{ \pm}-\Lambda^{ \pm}\right)=\mathrm{id}-\mathrm{id}=0$, it then follows that there exists a 2-chain $\lambda^{ \pm} \in \underline{\operatorname{hom}}\left(\mathfrak{L}_{\mathrm{pc} / \mathrm{fc}}(M), \mathfrak{L}_{\mathrm{pc} / \mathrm{fc}}(M)\right)_{2}$ such that $\widetilde{\Lambda}^{ \pm}-\Lambda^{ \pm}=\partial \lambda^{ \pm}$.

Remark 4.6. Lemma 4.5 (b) states that retarded/advanced trivializations are unique up to homotopy, provided they exist. From the proof of the lemma we see that even more is true and that such homotopies are unique up to higher homotopies. Indeed, if $\lambda^{ \pm}, \tilde{\lambda}^{ \pm} \in \underline{\operatorname{hom}}\left(\mathfrak{L}_{\mathrm{pc} / \mathrm{fc}}(M), \mathfrak{L}_{\mathrm{pc} / \mathrm{fc}}(M)\right)_{2}$ are 2-chains such that $\partial \tilde{\lambda}^{ \pm}=\widetilde{\Lambda}^{ \pm}-\Lambda^{ \pm}=\partial \lambda^{ \pm}$, then $\tilde{\lambda}^{ \pm}-\lambda^{ \pm}$is a 2 -cycle and hence, because of acyclicity of the mapping complex 
$\underline{\operatorname{hom}}\left(\mathfrak{L}_{\mathrm{pc} / \mathrm{fc}}(M), \mathfrak{L}_{\mathrm{pc} / \mathrm{fc}}(M)\right)$, there exists a 3-chain $\zeta^{ \pm} \in \underline{\operatorname{hom}}\left(\mathfrak{L}_{\mathrm{pc} / \mathrm{fc}}(M), \mathfrak{L}_{\mathrm{pc} / \mathrm{fc}}(M)\right)_{3}$ such that $\tilde{\lambda}^{ \pm}-\lambda^{ \pm}=\partial \zeta^{ \pm}$. The same argument applies to even higher homotopies, which implies that retarded/advanced trivializations are unique up to contractible choices.

Definition 4.7. A pair $\Lambda^{ \pm} \in \underline{\operatorname{hom}}\left(\mathfrak{L}_{\mathrm{pc} / \mathrm{fc}}(M), \mathfrak{L}_{\mathrm{pc} / \mathrm{fc}}(M)\right)_{1}$ of retarded/advanced trivializations is called compatible if the corresponding chain map $\Lambda: \mathfrak{L}(M) \rightarrow \operatorname{Sol}(M)$ from (4.8) satisfies the formal skew-adjointness property

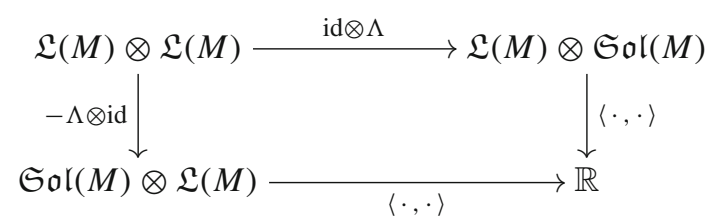

with respect to the integration pairings (4.2).

Definition 4.8. Suppose that $\Lambda^{ \pm} \in \underline{\operatorname{hom}}\left(\mathfrak{L}_{\mathrm{pc} / \mathrm{fc}_{\mathrm{c}}}(M), \mathfrak{L}_{\mathrm{pc} / \mathrm{fc}}(M)\right)_{1}$ is a compatible pair of retarded/advanced trivializations. The corresponding unshifted Poisson structure on the solution complex $\operatorname{Sol}(M)$ in (3.13) is the chain map $\tau: \mathfrak{L}(M) \otimes \mathfrak{L}(M) \rightarrow \mathbb{R}$ defined by the composition

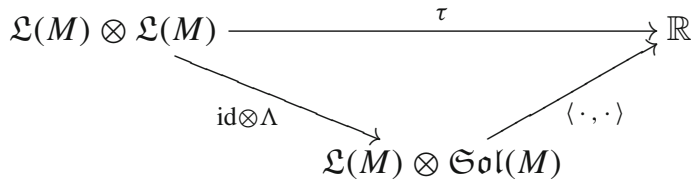

where $\mathfrak{L}(M)$ is the complex of linear observables (4.1) for $\operatorname{Sol}(M)$ and $\Lambda$ is given in (4.8).

Remark 4.9. Because $\Lambda^{ \pm} \in \underline{\operatorname{hom}}\left(\mathfrak{L}_{\mathrm{pc} / \mathrm{fc}}(M), \mathfrak{L}_{\mathrm{pc} / \mathrm{fc}}(M)\right)_{1}$ is by hypothesis a compatible pair (see Definition 4.7), it follows that the unshifted Poisson structure from Definition 4.8 is (graded) antisymmetric, i.e. $\tau \gamma=-\tau$ with $\gamma$ the symmetric braiding in $\mathbf{C h}_{\mathbb{R}}$. Hence, $\tau$ canonically defines a chain map (denoted with abuse of notation by the same symbol)

$$
\tau: \mathfrak{L}(M) \wedge \mathfrak{L}(M) \longrightarrow \mathbb{R}
$$

on the (graded) exterior product, or equivalently a 0 -cycle $\tau \in \underline{\operatorname{hom}}\left(\bigwedge^{2} \mathfrak{L}(M), \mathbb{R}\right)_{0}$ of the corresponding mapping complex. This perspective will be valuable below for studying homotopies between unshifted Poisson structures.

Corollary 4.10. Suppose that $\Lambda^{ \pm}, \widetilde{\Lambda}^{ \pm} \in \underline{\operatorname{hom}}\left(\mathfrak{L}_{\mathrm{pc} / \mathrm{fc}}(M), \mathfrak{L}_{\mathrm{pc} / \mathrm{fc}}(M)\right)_{1}$ are two compatible pairs of retarded/advanced trivializations and denote the corresponding unshifted Poisson structures by $\tau, \tilde{\tau} \in \underline{\operatorname{hom}}\left(\bigwedge^{2} \mathfrak{L}(M), \mathbb{R}\right)_{0}$. Then there exists a 1-chain $\rho \in$ $\underline{\operatorname{hom}}\left(\bigwedge^{2} \mathfrak{L}(M), \mathbb{R}\right)_{1}$ such that $\bar{\tau}-\tau=\partial \rho$. In particular, $[\tau]=[\tilde{\tau}]$ define the same homology class in $H_{0}\left(\underline{\operatorname{hom}}\left(\bigwedge^{2} \mathfrak{L}(M), \mathbb{R}\right)\right)$.

Proof. By Lemma 4.5 (b), there exists $\lambda^{ \pm} \in \underline{\operatorname{hom}}\left(\mathfrak{L}_{\mathrm{pc} / \mathrm{fc}}(M), \mathfrak{L}_{\mathrm{pc} / \mathrm{fc}}(M)\right)_{2}$ such that $\widetilde{\Lambda}^{ \pm}-\Lambda^{ \pm}=\partial \lambda^{ \pm}$, hence

$$
\tilde{\tau}-\tau=\partial\left(\langle\cdot, \cdot\rangle\left(\mathrm{id} \otimes\left(j_{\mathrm{pc}} \lambda^{+} \iota-j_{\mathrm{fc}} \lambda^{-} \iota\right)\right)\right)=: \partial \tilde{\rho} .
$$


Consider the decomposition $\tilde{\rho}=\widetilde{\rho}_{a}+\widetilde{\rho}_{s}=\frac{1}{2} \tilde{\rho}(\mathrm{id}-\gamma)+\frac{1}{2} \tilde{\rho}(\mathrm{id}+\gamma)$ of $\tilde{\rho}$ into its (graded) antisymmetric and symmetric parts. Because both $\tilde{\tau}$ and $\tau$ are (graded) antisymmetric, taking the (graded) antisymmetrization of (4.12) implies that $\widetilde{\tau}-\tau=\partial \widetilde{\rho}_{a}$ with the (graded) antisymmetric 1-chain $\widetilde{\rho}_{a} \in \underline{\operatorname{hom}}\left(\bigwedge^{2} \mathfrak{L}(M), \mathbb{R}\right)_{1}$.

Remark 4.11. We would like to emphasize that our Definition 4.8 of unshifted Poisson structures leaves one important question unanswered: Do compatible pairs of retarded/advanced trivializations exist? We do already know from Lemma 4.5 (b) that, provided they exist, retarded/advanced trivializations are unique up to homotopy, and so are their associated unshifted Poisson structures, see Corollary 4.10. Note that such questions are analogs of existence and uniqueness for Green's operators in ordinary field theory. We shall now investigate these issues in detail for Klein-Gordon and linear YangMills theory. This will in particular clarify the relationship between retarded/advanced trivializations and retarded/advanced Green's operators.

4.1. Klein-Gordon theory. Recall the Klein-Gordon solution complex $\mathfrak{S o l}^{\mathrm{KG}}(M)$ from Example 3.8. The corresponding complex of linear observables from Definition 4.1 then reads as

$$
\mathfrak{L}^{\mathrm{KG}}(M)=\left(0 \stackrel{0}{\longleftarrow} \Omega_{\mathrm{c}}^{(0)}(M) \stackrel{\square-m^{2}}{\longleftarrow} \Omega_{\mathrm{c}}^{0}(M) \stackrel{(1)}{\longleftarrow} 0\right) .
$$

Elements $\varphi \in \mathfrak{L}_{0}^{\mathrm{KG}}(M)=\Omega_{\mathrm{c}}^{0}(M)$ in degree 0 are interpreted as linear scalar field observables and elements $\alpha \in \mathfrak{L}_{1}^{\mathrm{KG}}(M)=\Omega_{\mathrm{c}}^{0}(M)$ in degree 1 as linear antifield observables. The evaluation of these observables on $\mathfrak{S o l}^{\mathrm{KG}}(M)$ is described by (4.2) and reads as

$$
\langle\varphi, \Phi\rangle=\int_{M} \varphi \Phi \operatorname{vol}_{M}, \quad\left\langle\alpha, \Phi^{\ddagger}\right\rangle=\int_{M} \alpha \Phi^{\ddagger} \operatorname{vol}_{M},
$$

for all $\Phi \in \mathfrak{S o r}_{0}^{\mathrm{KG}}(M)=\Omega^{0}(M)$ and all $\Phi^{\ddagger} \in \mathfrak{S o r}_{-1}^{\mathrm{KG}}(M)=\Omega^{0}(M)$. Note that only the zeroth homology of $\mathfrak{L}^{\mathrm{KG}}(M)$ is non-vanishing. It is given by the ordinary vector space $H_{0}\left(\mathfrak{L}^{\mathrm{KG}}(M)\right)=\Omega_{\mathrm{c}}^{0}(M) /\left(\square-m^{2}\right) \Omega_{\mathrm{c}}^{0}(M)$ of linear on-shell observables for Klein-Gordon theory, see e.g. [BDH13]. It follows that $\mathfrak{L}^{\mathrm{KG}}(M)$ is quasi-isomorphic to its zeroth homology $H_{0}\left(\mathfrak{L}^{\mathrm{KG}}(M)\right)$, regarded as a chain complex concentrated in degree 0 . In other words, for Klein-Gordon theory on $M$ it does not make any difference if we work with the complex of linear observables $\mathfrak{L}^{\mathrm{KG}}(M)$ or with the ordinary vector space $H_{0}\left(\mathfrak{L}^{\mathrm{KG}}(M)\right)$ of linear on-shell observables.

The shifted Poisson structure $\Upsilon^{\mathrm{KG}}: \mathfrak{L}^{\mathrm{KG}}(M) \otimes \mathfrak{L}^{\mathrm{KG}}(M) \rightarrow \mathbb{R}[1]$ from Definition 4.2 describes the following pairing between scalar field observables and antifield observables

$$
\Upsilon^{\mathrm{KG}}(\alpha, \varphi)=-\int_{M} \alpha \varphi \operatorname{vol}_{M}=\Upsilon^{\mathrm{KG}}(\varphi, \alpha),
$$

for all $\alpha \in \mathfrak{L}_{1}^{\mathrm{KG}}(M)=\Omega_{\mathrm{c}}^{0}(M)$ and $\varphi \in \mathfrak{L}_{0}^{\mathrm{KG}}(M)=\Omega_{\mathrm{c}}^{0}(M)$.

Our next aim is to classify all retarded/advanced trivializations in the sense of Definition 4.4 for Klein-Gordon theory. Recalling the complex $\mathfrak{L}^{\mathrm{KG}}(M)$ from (4.13), a 
retarded/advanced trivialization $\Lambda^{ \pm}$may be visualized by the down-right pointing arrows in the diagram

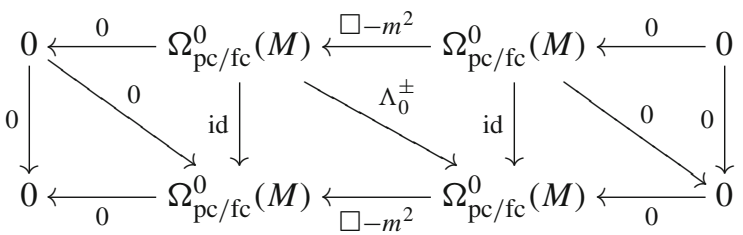

which in the present case are simply given by the data of a single linear map $\Lambda_{0}^{ \pm}$: $\Omega_{\mathrm{pc} / \mathrm{fc}}^{0}(M) \rightarrow \Omega_{\mathrm{pc} / \mathrm{fc}}^{0}(M)$. The condition id $=\partial \Lambda^{ \pm}$is equivalent to the two equalities

$$
\left(\square-m^{2}\right) \Lambda_{0}^{ \pm}=\mathrm{id}, \quad \Lambda_{0}^{ \pm}\left(\square-m^{2}\right)=\mathrm{id} .
$$

Proposition 4.12. For Klein-Gordon theory, there exists a unique retarded/advanced trivialization $\Lambda^{ \pm} \in \underline{\operatorname{hom}}\left(\mathfrak{L}_{\mathrm{pc} / \mathrm{fc}}^{\mathrm{KG}}(M), \mathfrak{L}_{\mathrm{pc} / \mathrm{fc}}^{\mathrm{KG}}(M)\right)_{1}$. It is given by the unique (extended) retarded/advanced Green's operator $\Lambda_{0}^{ \pm}=G^{ \pm}: \Omega_{\mathrm{pc} / \mathrm{fc}}^{0}(M) \rightarrow \Omega_{\mathrm{pc} / \mathrm{fc}}^{0}(M)$ for $\square-m^{2}$.

Proof. Recall from Sect. 2.1 that the (extended) retarded/advanced Green's operator $G^{ \pm}: \Omega_{\mathrm{pc} / \mathrm{fc}}^{0}(M) \rightarrow \Omega_{\mathrm{pc} / \mathrm{fc}}^{0}(M)$ for $\square-m^{2}$ satisfies (4.17) and hence setting $\Lambda_{0}^{ \pm}=G^{ \pm}$ defines a retarded/advanced trivialization $\Lambda^{ \pm} \in \underline{\operatorname{hom}}\left(\mathfrak{L}_{\mathrm{pc} / \mathrm{fc}}^{\mathrm{KG}}(M), \mathfrak{L}_{\mathrm{pc} / \mathrm{fc}}^{\mathrm{KG}}(M)\right)_{1}$. Uniqueness follows from Lemma 4.5 (b) and the fact that $\underline{\operatorname{hom}}\left(\mathfrak{L}_{\mathrm{pc} / \mathrm{fc}}^{\mathrm{KG}}(M), \mathfrak{L}_{\mathrm{pc} / \mathrm{fc}}^{\mathrm{KG}}(M)\right)_{2}=0$ is the zero vector space.

Because of (2.6), the unique retarded and advanced trivializations obtained above form a compatible pair in the sense of Definition 4.7. The corresponding unshifted Poisson structure from Definition 4.8 then reads as

$$
\tau^{\mathrm{KG}}\left(\varphi_{1}, \varphi_{2}\right)=\int_{M} \varphi_{1} G \varphi_{2} \operatorname{vol}_{M},
$$

for all $\varphi_{1}, \varphi_{2} \in \mathfrak{L}_{0}^{\mathrm{KG}}(M)=\Omega_{\mathrm{c}}^{0}(M)$, where $G:=G^{+}-G^{-}$is the causal propagator for $\square-m^{2}$. This is precisely the usual Poisson structure for Klein-Gordon theory, see e.g. [BDH13].

4.2. Linear Yang-Mills theory. Recall the linear Yang-Mills solution complex $\mathfrak{S o r}^{\mathrm{YM}}(M)$ from Example 3.9. The corresponding complex of linear observables from Definition 4.1 reads as

$$
\mathfrak{L}^{\mathrm{YM}}(M)=\left(\Omega_{\mathrm{c}}^{(-1)}(M) \stackrel{-\delta}{\longleftarrow} \Omega_{\mathrm{c}}^{1}(M) \stackrel{\delta \mathrm{d}}{\longleftarrow} \Omega_{\mathrm{c}}^{(1)}(M) \stackrel{-\mathrm{d}}{\longleftarrow} \Omega_{\mathrm{c}}^{(2)}(M)\right) .
$$

Elements $\varphi \in \mathfrak{L}_{0}^{\mathrm{YM}}(M)=\Omega_{\mathrm{c}}^{1}(M)$ in degree 0 are interpreted as linear gauge field observables and elements $\chi \in \mathfrak{L}_{-1}^{\mathrm{YM}}(M)=\Omega_{\mathrm{c}}^{0}(M)$ in degree -1 as linear ghost field observables. Elements $\alpha \in \mathfrak{L}_{1}^{\mathrm{YM}}(M)=\Omega_{\mathrm{c}}^{1}(M)$ in degree 1 and $\beta \in \mathfrak{L}_{2}^{\mathrm{YM}}(M)=$ $\Omega_{\mathrm{c}}^{0}(M)$ in degree 2 are interpreted as linear observables for the antifields $A^{\ddagger}$ and $c^{\ddagger}$. The evaluation of these observables on $\mathfrak{S o r}^{\mathrm{YM}}(M)$ is described by (4.2) and reads as

$$
\langle\varphi, A\rangle=\int_{M} \varphi \wedge * A, \quad\langle\chi, c\rangle=\int_{M} \chi c \operatorname{vol}_{M},
$$




$$
\left\langle\alpha, A^{\ddagger}\right\rangle=\int_{M} \alpha \wedge * A^{\ddagger}, \quad\left\langle\beta, c^{\ddagger}\right\rangle=\int_{M} \beta c^{\ddagger} \operatorname{vol}_{M},
$$

for all gauge fields $A \in \mathfrak{S o r}_{0}^{\mathrm{YM}}(M)=\Omega^{1}(M)$, ghost fields $c \in \mathfrak{S o r}_{1}^{\mathrm{YM}}(M)=\Omega^{0}(M)$ and antifields $A^{\ddagger} \in \operatorname{Sor}_{-1}^{\mathrm{YM}}(M)=\Omega^{1}(M)$ and $c^{\ddagger} \in \operatorname{Sor}_{-2}^{\mathrm{YM}}(M)=\Omega^{0}(M)$. The homologies of $\mathfrak{L}^{\mathrm{YM}}(M)$ can be computed explicitly and admit a physical interpretation, see also Example 3.9.

- $H_{-1}\left(\mathfrak{L}^{\mathrm{YM}}(M)\right)=H_{\mathrm{c}, \delta}^{0}(M) \cong H_{\mathrm{c}, \mathrm{dR}}^{m}(M)$ is by Poincaré duality the linear dual of the vector space $H_{1}\left(\mathfrak{S o l}^{\mathrm{YM}}(M)\right) \cong H_{\mathrm{dR}}^{0}(M)$, i.e. it consists of linear observables testing those ghost fields that act trivially on gauge fields.

- $H_{0}\left(\mathfrak{L}^{\mathrm{YM}}(M)\right)=\Omega_{\mathrm{c}, \delta}^{1}(M) / \delta \mathrm{d} \Omega_{\mathrm{c}}^{1}(M)$ is the usual vector space of linear gaugeinvariant on-shell observables, see e.g. [SDH14,BDS14,BDHS14,FL16, Ben16, BSS16].

- $H_{1}\left(\mathfrak{L}^{\mathrm{YM}}(M)\right)=\Omega_{\mathrm{c}, \delta \mathrm{d}}^{1}(M) / \mathrm{d} \Omega_{\mathrm{c}}^{0}(M) \cong H_{\mathrm{c}, \mathrm{dR}}^{1}(M)$ is by Poincaré duality the linear dual of the vector space $H_{-1}\left(\mathfrak{S o r}^{\mathrm{YM}}(M)\right) \cong H_{\mathrm{dR}}^{m-1}(M)$, i.e. it consists of linear observables testing obstructions to solving the inhomogeneous linear Yang-Mills equation $\delta \mathrm{d} A=j$ with $j \in \Omega_{\delta}^{1}(M)$.

- $H_{2}\left(\mathfrak{L}^{\mathrm{YM}}(M)\right)=H_{\mathrm{c}, \mathrm{dR}}^{0}(M) \cong 0$, because $M \cong \mathbb{R} \times \Sigma$.

The shifted Poisson structure $\Upsilon^{\mathrm{YM}}: \mathfrak{L}^{\mathrm{YM}}(M) \otimes \mathfrak{L}^{\mathrm{YM}}(M) \rightarrow \mathbb{R}[1]$ from Definition 4.2 describes the following pairing between gauge or respectively ghost field observables and their corresponding antifield observables

$$
\begin{aligned}
& \Upsilon^{\mathrm{YM}}(\alpha, \varphi)=-\int_{M} \alpha \wedge * \varphi=\Upsilon^{\mathrm{YM}}(\varphi, \alpha), \\
& \Upsilon^{\mathrm{YM}}(\beta, \chi)=\int_{M} \beta \chi \operatorname{vol}_{M}=\Upsilon^{\mathrm{YM}}(\chi, \beta),
\end{aligned}
$$

for all $\varphi \in \mathfrak{L}_{0}^{\mathrm{YM}}(M)=\Omega_{\mathrm{c}}^{1}(M), \alpha \in \mathfrak{L}_{1}^{\mathrm{YM}}(M)=\Omega_{\mathrm{c}}^{1}(M), \chi \in \mathfrak{L}_{-1}^{\mathrm{YM}}(M)=\Omega_{\mathrm{c}}^{0}(M)$ and $\beta \in \mathfrak{L}_{2}^{\mathrm{YM}}(M)=\Omega_{\mathrm{c}}^{0}(M)$.

We now construct a compatible pair of retarded/advanced trivializations in the sense of Definitions 4.4 and 4.7 for linear Yang-Mills theory. Recalling the complex $\mathfrak{L}^{\mathrm{YM}}(M)$ from (4.19), a retarded/advanced trivialization $\Lambda^{ \pm}$may be visualized by the down-right pointing arrows in the diagram

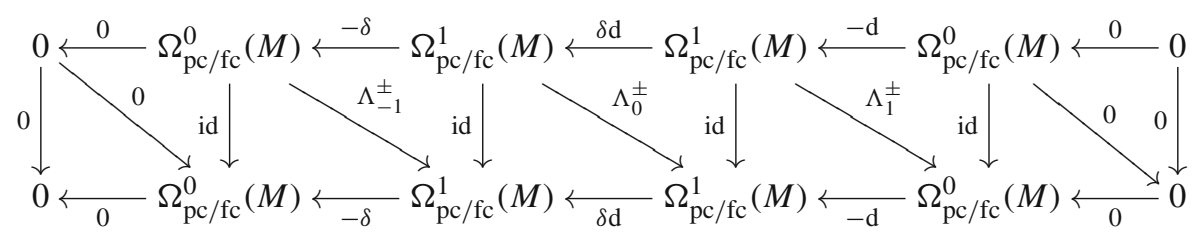

which in the present case are three linear maps $\Lambda_{-1}^{ \pm}: \Omega_{\mathrm{pc} / \mathrm{fc}}^{0}(M) \rightarrow \Omega_{\mathrm{pc} / \mathrm{fc}}^{1}(M), \Lambda_{0}^{ \pm}$: $\Omega_{\mathrm{pc} / \mathrm{fc}}^{1}(M) \rightarrow \Omega_{\mathrm{pc} / \mathrm{fc}}^{1}(M)$ and $\Lambda_{1}^{ \pm}: \Omega_{\mathrm{pc} / \mathrm{fc}}^{1}(M) \rightarrow \Omega_{\mathrm{pc} / \mathrm{fc}}^{0}(M)$, subject to the four identities

$$
-\delta \Lambda_{-1}^{ \pm}=\mathrm{id}, \quad \delta \mathrm{d} \Lambda_{0}^{ \pm}-\Lambda_{-1}^{ \pm} \delta=\mathrm{id}, \quad \Lambda_{0}^{ \pm} \delta \mathrm{d}-\mathrm{d} \Lambda_{1}^{ \pm}=\mathrm{id}, \quad-\Lambda_{1}^{ \pm} \mathrm{d}=\mathrm{id} .
$$


Proposition 4.13. Denote by $G^{ \pm}: \Omega_{\mathrm{pc} / \mathrm{fc}}^{1}(M) \rightarrow \Omega_{\mathrm{pc} / \mathrm{fc}}^{1}(M)$ the (extended) retarded/ advanced Green's operators for the d'Alembert operator $\square: \Omega^{1}(M) \rightarrow \Omega^{1}(M)$ on 1-forms. The choices

$$
\Lambda_{-1}^{ \pm}=-G^{ \pm} \mathrm{d}, \quad \Lambda_{0}^{ \pm}=G^{ \pm}, \quad \Lambda_{1}^{ \pm}=-\delta G^{ \pm}
$$

define a compatible pair of retarded/advanced trivializations for linear Yang-Mills theory.

Proof. This follows immediately from the properties of Green's operators stated in Sect. 2.1, see in particular Example 2.1.

Remark 4.14. In contrast to the example of Klein-Gordon theory from Sect. 4.1, the retarded/advanced trivializations of linear Yang-Mills theory are not unique, but only unique up to contractible choices, see Lemma 4.5 and Remark 4.6. Any other retarded/advanced trivialization $\widetilde{\Lambda}^{ \pm}$differs from our $\Lambda^{ \pm}$above by the differential of a 2-chain $\lambda^{ \pm} \in \underline{\operatorname{hom}}\left(\mathfrak{L}_{\mathrm{pc} / \mathrm{fc}}^{\mathrm{YM}}(M), \mathfrak{L}_{\mathrm{pc} / \mathrm{fc}}(M)\right)_{2}$. Explicitly, the three non-zero components of $\widetilde{\Lambda}^{ \pm}$read as

$$
\begin{array}{rll}
\widetilde{\Lambda}_{-1}^{ \pm}=-G^{ \pm} \mathrm{d}+\delta \mathrm{d} \lambda_{-1}^{ \pm} & : & \Omega_{\mathrm{pc} / \mathrm{fc}}^{0}(M) \longrightarrow \Omega_{\mathrm{pc} / \mathrm{fc}}^{1}(M), \\
\widetilde{\Lambda}_{0}^{ \pm}=G^{ \pm}-\mathrm{d} \lambda_{0}^{ \pm}+\lambda_{-1}^{ \pm} \delta: & \Omega_{\mathrm{pc} / \mathrm{fc}}^{1}(M) \longrightarrow \Omega_{\mathrm{pc} / \mathrm{fc}}^{1}(M), \\
\widetilde{\Lambda}_{1}^{ \pm}=-\delta G^{ \pm}-\lambda_{0}^{ \pm} \delta \mathrm{d} & : & \Omega_{\mathrm{pc} / \mathrm{fc}}^{1}(M) \longrightarrow \Omega_{\mathrm{pc} / \mathrm{fc}}^{0}(M),
\end{array}
$$

where $\lambda_{-1}^{ \pm}: \Omega_{\mathrm{pc} / \mathrm{fc}}^{0}(M) \rightarrow \Omega_{\mathrm{pc} / \mathrm{fc}}^{1}(M)$ and $\lambda_{0}^{ \pm}: \Omega_{\mathrm{pc} / \mathrm{fc}}^{1}(M) \rightarrow \Omega_{\mathrm{pc} / \mathrm{fc}}^{0}(M)$ are the two non-zero components of the 2-chain $\lambda^{ \pm}$.

The unshifted Poisson structure (see Definition 4.8) that corresponds to our compatible pair of retarded/advanced trivializations $\Lambda^{ \pm}$from Proposition 4.13 reads as

$$
\begin{aligned}
\tau^{\mathrm{YM}}\left(\varphi_{1}, \varphi_{2}\right) & =\int_{M} \varphi_{1} \wedge * G \varphi_{2}=-\tau^{\mathrm{YM}}\left(\varphi_{2}, \varphi_{1}\right), \\
\tau^{\mathrm{YM}}(\alpha, \chi) & =-\int_{M} \alpha \wedge * G \mathrm{~d} \chi=\tau^{\mathrm{YM}}(\chi, \alpha),
\end{aligned}
$$

for all $\varphi_{1}, \varphi_{2} \in \mathfrak{L}_{0}^{\mathrm{YM}}(M)=\Omega_{\mathrm{c}}^{1}(M), \alpha \in \mathfrak{L}_{1}^{\mathrm{YM}}(M)=\Omega_{\mathrm{c}}^{1}(M)$ and $\chi \in \mathfrak{L}_{-1}^{\mathrm{YM}_{(}}(M)=$ $\Omega_{\mathrm{c}}^{0}(M)$, where $G:=G^{+}-G^{-}$is the causal propagator for the d'Alembert operator $\square$ on 1-forms. Note that this Poisson structure acts non-trivially on pairs $\left(\varphi_{1}, \varphi_{2}\right)$ of linear gauge field observables and also non-trivially on pairs $(\alpha, \chi)$ consisting of a linear antifield observable $\alpha$ and a linear ghost field observable $\chi$. It extends to the richer level of chain complexes of linear observables $\mathfrak{L}^{\mathrm{YM}}(M)$ the usual Poisson structure on linear gauge-invariant on-shell observables $H_{0}\left(\mathfrak{L}^{\mathrm{YM}}(M)\right)$, see e.g. [SDH14, BDS14, BDHS14, FL16,Ben16,BSS16].

To conclude this section, we would like to emphasize that any other choice of a compatible pair of retarded/advanced trivializations $\widetilde{\Lambda}^{ \pm}$(see Remark 4.14 for a concrete description) defines an unshifted Poisson structure $\tilde{\tau}=\tau^{\mathrm{YM}}+\partial \rho$ that agrees with (4.26) up to homotopy, see Corollary 4.10. We shall prove in Proposition 5.3 that the quantization of two homotopic Poisson structures yields quasi-isomorphic observable algebras, i.e. the quasi-isomorphism type of the resulting quantum theory depends only on the uniquely defined homology classes $\left[\Lambda^{ \pm}\right] \in H_{1}\left(\underline{\operatorname{hom}}\left(\mathfrak{L}_{\mathrm{pc} / \mathrm{fc}}^{\mathrm{YM}}(M), \mathfrak{L}_{\mathrm{pc} / \mathrm{fc}}^{\mathrm{YM}}(M)\right)\right)$. 


\section{Quantization}

The goal of this section is to develop a chain complex analog of the usual canonical commutation relations (CCR) quantization of vector spaces endowed with Poisson structures, see e.g. [BDH13]. The input of our construction is a pair $(V, \tau)$ consisting of a chain complex $V \in \mathbf{C h}_{\mathbb{R}}$ and a chain map $\tau: V \wedge V \rightarrow \mathbb{R}$. We shall call $(V, \tau)$ an unshifted Poisson complex. The output of our construction is a differential graded unital and associative $*$-algebra $\mathfrak{C} \mathfrak{C}(V, \tau)$ over the field of complex numbers $\mathbb{C}$ that implements the canonical commutation relations determined by $\tau$. We shall investigate homotopical properties of this quantization prescription and in particular prove that, up to quasi-isomorphism, the quantization $\mathfrak{C} \mathfrak{R}(V, \tau)$ does only depend on the quasiisomorphism type of $(V, \tau)$ and on the homology class $[\tau] \in H_{0}\left(\operatorname{hom}\left(\bigwedge^{2} V, \mathbb{R}\right)\right)$ of $\tau$. In the context of our examples from Sect. 4, this means that both Klein-Gordon theory and linear Yang-Mills theory can be consistently quantized by our methods.

Let us now explain in some detail the CCR quantization $\mathfrak{C} \mathfrak{R}(V, \tau)$ of an unshifted Poisson complex $(V, \tau)$. We denote by $T_{\mathbb{C}}^{\otimes} V$ the free differential graded unital and associative $*$-algebra generated by $V \in \mathbf{C h}_{\mathbb{R}}$. Concretely, $T_{\mathbb{C}}^{\otimes} V$ is given by

$$
T_{\mathbb{C}}^{\otimes} V:=\bigoplus_{n=0}^{\infty} V_{\mathbb{C}}^{\otimes n}
$$

where $V_{\mathbb{C}}:=V \otimes \mathbb{C} \in \mathbf{C h}_{\mathbb{C}}$ is the complexification of $V$, together with the usual multiplication $\mu: T_{\mathbb{C}}^{\otimes} V \otimes T_{\mathbb{C}}^{\otimes} V \rightarrow T_{\mathbb{C}}^{\otimes} V$ and unit $\eta: \mathbb{C} \rightarrow T_{\mathbb{C}}^{\otimes} V$ determined by $\mu\left(\left(v_{1} \otimes \cdots \otimes v_{n}\right) \otimes\left(v_{1}^{\prime} \otimes \cdots \otimes v_{m}^{\prime}\right)\right)=v_{1} \otimes \cdots \otimes v_{n} \otimes v_{1}^{\prime} \otimes \cdots \otimes v_{m}^{\prime}$, for all $v_{1}, \ldots, v_{n}, v_{1}^{\prime}, \ldots, v_{m}^{\prime} \in V$, and $\mathbb{1}:=\eta(1)=1 \in V_{\mathbb{C}}^{\otimes 0}=\mathbb{C}$. The $\mathbb{C}$-antilinear $*-$ involution is determined by $v^{*}=v$, for all $v \in V$. The CCR quantization is defined as the quotient

$$
\mathfrak{C C R}(V, \tau):=T_{\mathbb{C}}^{\otimes} V / \mathcal{I}_{(V, \tau)}
$$

by the two-sided differential graded $*$-ideal $\mathcal{I}_{(V, \tau)} \subseteq T_{\mathbb{C}}^{\otimes} V$ generated by the (graded) canonical commutation relations

$$
v_{1} \otimes v_{2}-(-1)^{\left|v_{1}\right|\left|v_{2}\right|} v_{2} \otimes v_{1}=i \tau\left(v_{1}, v_{2}\right) \mathbb{1},
$$

for all homogeneous elements $v_{1}, v_{2} \in V$ with degrees denoted by $\left|v_{1}\right|,\left|v_{2}\right| \in \mathbb{Z}$. We note that CCR quantization is functorial

$$
\mathfrak{C C} \Re: \operatorname{PoCh}_{\mathbb{R}} \longrightarrow \operatorname{dg}^{*} \mathrm{Alg}_{\mathbb{C}}
$$

for the following natural choices of categories:

- $\mathbf{P o C h}_{\mathbb{R}}$ denotes the category of unshifted Poisson complexes, i.e. objects are pairs $(V, \tau)$ consisting of a chain complex $V \in \mathbf{C h}_{\mathbb{R}}$ and a chain map $\tau: V \wedge V \rightarrow \mathbb{R}$ and morphisms $f:(V, \tau) \rightarrow\left(V^{\prime}, \tau^{\prime}\right)$ are chain maps $f: V \rightarrow V^{\prime}$ preserving the Poisson structures $\tau^{\prime}(f \wedge f)=\tau$.

- $\mathbf{d g}^{*} \mathbf{A l g}_{\mathbb{C}}$ denotes the usual category of differential graded unital and associative $*$-algebras. 
Remark 5.1. Every ordinary Poisson vector space $(V, \tau)$ defines an unshifted Poisson complex whose underlying chain complex is concentrated in degree 0 . In such cases our CCR quantization $\mathfrak{C} \mathfrak{C} \Re(V, \tau)$ yields a differential graded $*$-algebra concentrated in degree 0, which coincides with the usual CCR algebra from the non-homotopical framework, see e.g. [BDH13].

For our homotopical analysis of CCR quantization, we endow both $\mathbf{P o C h} \mathbf{h}_{\mathbb{R}}$ and $\mathbf{d g}^{*} \mathbf{A} \mathbf{I g}_{\mathbb{C}}$ with the structure of a homotopical category in the sense of [DHKS04,Rie14]. This is a more flexible framework than model category theory, which is very convenient for our purposes because $\mathbf{P o C h} \mathbf{C h}_{\mathbb{R}}$ is not a model category as it is not cocomplete. Similarly to model category theory, a homotopical category is a category with a choice of weak equivalences (containing all isomorphisms and satisfying the so-called 2-of-6 property), however there is no need to introduce compatible classes of fibrations and cofibrations or to require the category to be bicomplete. In our context, we introduce the following canonical homotopical category structures on $\mathbf{P o C h}_{\mathbb{R}}$ and $\mathbf{d g}^{*} \mathbf{A l g} \mathbf{C}_{\mathbb{C}}$.

Definition 5.2. (i) A morphism $f:(V, \tau) \rightarrow\left(V^{\prime}, \tau^{\prime}\right)$ in $\mathbf{P o C h}_{\mathbb{R}}$ is a weak equivalence if its underlying chain map $f: V \rightarrow V^{\prime}$ is a quasi-isomorphism in $\mathbf{C h}_{\mathbb{R}}$.

(ii) A morphism $\kappa: A \rightarrow A^{\prime}$ in $\mathbf{~ d g}^{*} \mathbf{A} \mathbf{I g}_{\mathbb{C}}$ is a weak equivalence if its underlying chain map is a quasi-isomorphism in $\mathbf{C h}_{\mathbb{C}}$.

The next result shows that the CCR functor (5.4) has very pleasant homotopical properties, which in particular ensure that our examples of linear gauge theories from Sect. 4 can be quantized consistently. The proof of the following proposition is slightly technical and hence it will be carried out in detail in "Appendix A".

Proposition 5.3. (a) The CCR functor (5.4) is a homotopical functor, i.e. it preserves the weak equivalences introduced in Definition 5.2.

(b) Let $(V, \tau) \in \mathbf{P o C h}_{\mathbb{R}}$ be an unshifted Poisson complex and $\rho \in \underline{\operatorname{hom}}\left(\bigwedge^{2} V, \mathbb{R}\right)_{1}$ a 1-chain. Then there exists a zig-zag

$$
\mathfrak{C C R}(V, \tau) \stackrel{\sim}{\longleftarrow} A_{(V, \tau, \rho)} \stackrel{\sim}{\longrightarrow} \mathfrak{C C} \mathfrak{R}(V, \tau+\partial \rho)
$$

\section{of weak equivalences in $\mathbf{d g}^{*} \mathbf{A} \mathbf{I g}_{\mathbb{C}}$.}

In our context of linear gauge theories from Sect. 4, we immediately obtain the following crucial result as a direct consequence of Proposition 5.3 and Corollary 4.10.

Corollary 5.4. Suppose that $\Lambda^{ \pm}, \widetilde{\Lambda}^{ \pm} \in \underline{\operatorname{hom}}\left(\mathfrak{L}_{\mathrm{pc} / \mathrm{fc}}(M), \mathfrak{L}_{\mathrm{pc} / \mathrm{fc}}(M)\right)_{1}$ are two compatible pairs of retarded/advanced trivializations and denote the corresponding unshifted Poisson structures by $\tau, \tilde{\tau}: \mathfrak{L}(M) \wedge \mathfrak{L}(M) \rightarrow \mathbb{R}$. Then the two CCR quantizations $\mathfrak{C C} \mathfrak{R}(\mathfrak{L}(M), \tau) \simeq \mathfrak{C} \mathfrak{C}(\mathfrak{L}(M), \tilde{\tau})$ are equivalent via a zig-zag of weak equivalences in $\operatorname{dg}^{*} \operatorname{Alg}_{\mathbb{C}}$

Example 5.5. Recall from Sect. 4.1 the unshifted Poisson complex $\left(\mathfrak{L}^{\mathrm{KG}}(M), \tau^{\mathrm{KG}}\right) \in$ $\mathbf{P o C h}_{\mathbb{R}}$ for Klein-Gordon theory on an oriented and time-oriented globally hyperbolic Lorentzian manifold $M$, see in particular (4.13) and (4.18). Observe that the quotient map $\mathfrak{L}^{\mathrm{KG}}(M) \rightarrow H_{0}\left(\mathfrak{L}^{\mathrm{KG}}(M)\right)=\Omega_{\mathrm{c}}^{0}(M) /\left(\square-m^{2}\right) \Omega_{\mathrm{c}}^{0}(M)$ to the vector space of linear on-shell observables (regarded as a chain complex concentrated in degree 0 ) is a quasi-isomorphism and that the unshifted Poisson structure (4.18) descends to the quotient because of $G\left(\square-m^{2}\right)=0$. Hence, we obtain a weak equivalence $\left(\mathfrak{L}^{\mathrm{KG}}(M), \tau^{\mathrm{KG}}\right) \stackrel{\sim}{\rightarrow}\left(H_{0}\left(\mathfrak{L}^{\mathrm{KG}}(M)\right), \tau^{\mathrm{KG}}\right)$ in $\mathbf{P o C h}_{\mathbb{R}}$ from our original unshifted Poisson complex to an unshifted Poisson complex concentrated in degree 0 , which is simply 
the ordinary Poisson vector space of linear on-shell observables. As a consequence of Proposition 5.3 (a), it follows that our CCR quantization $\mathfrak{C} \mathfrak{C} \mathfrak{R}\left(\mathfrak{L}^{\mathrm{KG}}(M), \tau^{\mathrm{KG}}\right)$ is weakly equivalent in $\mathbf{d g}^{*} \mathbf{A l g} \mathbb{C}_{\mathbb{C}}$ to the ordinary CCR quantization $\mathfrak{C} \mathfrak{C} \mathfrak{R}\left(H_{0}\left(\mathfrak{L}^{\mathrm{KG}}(M)\right), \tau^{\mathrm{KG}}\right)$ of Klein-Gordon theory as a unital and associative $*$-algebra (regarded as a differential graded $*$-algebra concentrated in degree 0 ).

Example 5.6. Recall from Sect. 4.2 the unshifted Poisson complex $\left(\mathfrak{L}^{\mathrm{YM}}(M), \tau^{\mathrm{YM}}\right) \in$ $\mathbf{P o C h}_{\mathbb{R}}$ for linear Yang-Mills theory on an oriented and time-oriented globally hyperbolic Lorentzian manifold $M$, see in particular (4.19) and (4.26). As a consequence of Corollary 5.4, the CCR quantization $\mathfrak{C} \mathfrak{C} \mathfrak{R}\left(\mathfrak{L}^{\mathrm{YM}}(M), \tau^{\mathrm{YM}}\right)$ for our particular choice of retarded/advanced trivializations given in Proposition 4.13 is equivalent via a zig-zag of weak equivalences in $\mathbf{d g}^{*} \mathbf{A l g} \mathbb{C}_{\mathbb{C}}$ to the CCR quantization $\mathfrak{C} \mathfrak{C} \mathfrak{R}\left(\mathfrak{L}^{\mathrm{YM}}(M), \tilde{\tau}=\tau^{\mathrm{YM}}+\partial \rho\right)$ for any other choice, see also Remark 4.14. Thus, we obtain a consistent quantization prescription for linear Yang-Mills theory.

To be very explicit, let us also write out the (graded) commutation relations of the generators of $\mathfrak{C} \mathfrak{C} \mathfrak{R}\left(\mathfrak{L}^{\mathrm{YM}}(M), \tau^{\mathrm{YM}}\right)$. We use a suggestive notation and denote the smeared linear quantum observables for gauge fields by $\widehat{A}(\varphi)$, for $\varphi \in \mathfrak{L}_{0}^{\mathrm{YM}}(M)=\Omega_{\mathrm{c}}^{1}(M)$, the ones for ghost fields by $\widehat{c}(\chi)$, for $\chi \in \mathfrak{L}_{-1}^{\mathrm{YM}}(M)=\Omega_{\mathrm{c}}^{0}(M)$, and the ones for antifields by $\widehat{A}^{\ddagger}(\alpha)$ and $\widehat{c}^{\ddagger}(\beta)$, for $\alpha \in \mathfrak{L}_{1}^{\mathrm{YM}}(M)=\Omega_{\mathrm{c}}^{1}(M)$ and $\beta \in \mathfrak{L}_{2}^{\mathrm{YM}}(M)=\Omega_{\mathrm{c}}^{0}(M)$. Then (4.26) and (5.3) yield the following non-vanishing (graded) commutation relations

$$
\begin{aligned}
{\left[\widehat{A}\left(\varphi_{1}\right), \widehat{A}\left(\varphi_{2}\right)\right] } & =i \int_{M} \varphi_{1} \wedge * G \varphi_{2} \mathbb{1}, \\
{\left[\widehat{A}^{\ddagger}(\alpha), \widehat{c}(\chi)\right] } & =-i \int_{M} \alpha \wedge * G \mathrm{~d} \chi \mathbb{1}=\left[\widehat{c}(\chi), \widehat{A}^{\ddagger}(\alpha)\right],
\end{aligned}
$$

for all $\varphi_{1}, \varphi_{2} \in \mathfrak{L}_{0}^{\mathrm{YM}}(M)=\Omega_{\mathrm{c}}^{1}(M), \alpha \in \mathfrak{L}_{1}^{\mathrm{YM}}(M)=\Omega_{\mathrm{c}}^{1}(M)$ and $\chi \in \mathfrak{L}_{-1}^{\mathrm{YM}}(M)=$ $\Omega_{\mathrm{c}}^{0}(M)$.

\section{Functoriality and Homotopy AQFT Axioms}

Our results and constructions in the previous sections considered a fixed oriented and time-oriented globally hyperbolic Lorentzian manifold $M$. In order to obtain an algebraic quantum field theory (AQFT), in the original sense of Haag and Kastler [HK64] or in the more modern sense of Brunetti, Fredenhagen and Verch [BFV03], we have to analyze functoriality of our constructions with respect to a suitable class of spacetime embeddings $f: M \rightarrow N$. The relevant categories are defined as follows:

- Loc denotes the category of oriented and time-oriented globally hyperbolic Lorentzian manifolds (of a fixed dimension $m \geq 2$ ) with morphisms $f: M \rightarrow N$ given by all orientation and time-orientation preserving isometric embeddings whose image $f(M) \subseteq N$ is open and causally convex.

- For any $\bar{M} \in \mathbf{L o c}$, we denote by Loc $/ \bar{M}$ the corresponding slice category. Its objects are all Loc-morphisms $m: M \rightarrow \bar{M}$ with target $\bar{M}$ and its morphisms $f:(m: M \rightarrow \bar{M}) \rightarrow(n: N \rightarrow \bar{M})$ are all commutative triangles

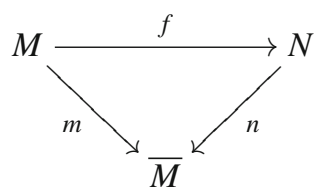


in Loc. Note that Loc $/ \bar{M} \simeq \operatorname{COpen}(\bar{M})$ is equivalent to the category of all causally convex open subsets $U \subseteq \bar{M}$ with morphisms given by subset inclusion.

As observed in Examples 5.5 and 5.6, our homotopy theoretical constructions naturally define differential graded $*$-algebras of quantum observables for each spacetime $M$. As a consequence, the relevant variants of AQFT to describe such models should take values in the model category $\mathbf{C h}_{\mathbb{C}}$ of chain complexes in contrast to the usual category $\mathbf{V e c}_{\mathbb{C}}$ of vector spaces. Based on the recent operadic approach to AQFT [BSW17,BSW19a], such algebraic structures were systematically investigated in [BSW19b]. One of the outcomes of these studies is a concept of homotopy AQFTs, i.e. homotopy-coherent AQFTs that are obtained by a resolution of the relevant operad. Since in the present paper our ground field $\mathbb{C}$ has characteristic 0, the strictification theorem of [BSW19b] implies that every homotopy AQFT can be strictified and hence all possible variants of homotopy AQFT are equivalent. To describe our concrete examples in the present paper, it is sufficient and very convenient to consider the following semi-strict model for homotopy AQFTs, where both functoriality and Einstein causality hold strictly, but the time-slice axiom is replaced by an appropriate homotopical analog.

Definition 6.1 A (semi-strict) homotopy AQFT on Loc is a functor $\mathfrak{A}: \mathbf{L o c} \rightarrow \mathbf{d g}^{*} \mathbf{A l g} \mathbb{C}_{\mathbb{C}}$ such that the following hold true:

(i) Strict Einstein causality axiom: For every pair $\left(f_{1}: M_{1} \rightarrow N, f_{2}: M_{2} \rightarrow N\right)$ of Loc-morphisms with causally disjoint images, the chain map

$$
\left[\mathfrak{A}\left(f_{1}\right)(-), \mathfrak{A}\left(f_{2}\right)(-)\right]: \mathfrak{A}\left(M_{1}\right) \otimes \mathfrak{A}\left(M_{2}\right) \longrightarrow \mathfrak{A}(N)
$$

is zero, where $[-,-]:=\mu-\mu \gamma: \mathfrak{A}(N) \otimes \mathfrak{A}(N) \rightarrow \mathfrak{A}(N)$ denotes the (graded) commutator in $\mathfrak{A}(N)$.

(ii) Homotopy time-slice axiom: For every Cauchy morphism, i.e. a Loc-morphism $f$ : $M \rightarrow N$ such that the image $f(M) \subseteq N$ contains a Cauchy surface of $N$, the map $\mathfrak{A}(f): \mathfrak{A}(M) \stackrel{\sim}{\rightarrow} \mathfrak{A}(N)$ is a weak equivalence in $\mathbf{d g}^{*} \mathbf{A} \mathbf{l g}_{\mathbb{C}}$.

For any $\bar{M} \in \mathbf{L o c}$, a homotopy $A Q F T$ on $\bar{M}$ is a functor $\mathfrak{A}: \mathbf{L o c} / \bar{M} \rightarrow \mathbf{d g}^{*} \mathbf{A l g}_{\mathbb{C}}$ on the slice category such that the evident analogs of Einstein causality and time-slice hold true.

Remark 6.2. Homotopy AQFTs on Loc are chain complex analogs of theories in the sense of Brunetti, Fredenhagen and Verch [BFV03], while homotopy AQFTs on a fixed $\bar{M} \in$ Loc are chain complex analogs of theories in the sense of Haag and Kastler [HK64]. Note that every homotopy AQFT $\mathfrak{A}:$ Loc $\rightarrow \mathbf{d g}^{*} \mathbf{A l g}_{\mathbb{C}}$ on Loc defines a homotopy AQFT $\mathfrak{A}_{\bar{M}}:=\mathfrak{A}_{\bar{M}}: \mathbf{L o c} / \bar{M} \rightarrow \mathbf{d g}^{*} \mathbf{A} \mathbf{l g}_{\mathbb{C}}$ on every $\bar{M} \in \mathbf{L o c}$ via precomposition with the forgetful functor $\mathfrak{U}_{\bar{M}}:$ Loc $/ \bar{M} \rightarrow$ Loc. Explicitly, the latter is given on objects by $(m: M \rightarrow \bar{M}) \mapsto M$ and on morphisms by $(f:(m: M \rightarrow \bar{M}) \rightarrow(n: N \rightarrow$ $\bar{M})) \mapsto(f: M \rightarrow N)$.

In the following let us assume that, in the context of Definition 3.1, the vector bundles $F_{n} \rightarrow M$ with fiber metrics $h_{n}$ and also the linear differential operator $Q$ are natural on Loc. Using the associated pullbacks $f^{*}: \mathfrak{F}_{n}(N) \rightarrow \mathfrak{F}_{n}(M)$ of sections along Locmorphisms $f: M \rightarrow N$, this implies that the assignment $M \mapsto \mathfrak{F}(M)$ of field complexes (3.1) is contravariantly functorial, i.e.

$$
\mathfrak{F}: \mathbf{L o c}^{\mathrm{op}} \longrightarrow \mathbf{C h}_{\mathbb{R}}
$$


Assuming further that the action (3.7) (or equivalently the linear differential operator $P$ ) is natural implies that the assignment $M \mapsto \operatorname{Sol}(M)$ of solution complexes (3.13) is contravariantly functorial too, i.e.

$$
\text { Sol }: \text { Loc }^{\text {op }} \longrightarrow \mathbf{C h}_{\mathbb{R}} \text {. }
$$

Using also pushforwards $f_{*}: \mathfrak{F}_{n, \mathrm{c}}(M) \rightarrow \mathfrak{F}_{n, \mathrm{c}}(N)$ of compactly supported sections along Loc-morphisms $f: M \rightarrow N$, one observes that the assignment $M \mapsto \mathfrak{L}(M)$ of complexes of linear observables (4.1) is covariantly functorial, i.e.

$$
\mathfrak{L}: \mathbf{L o c} \longrightarrow \mathbf{C h}_{\mathbb{R}},
$$

and that the integration pairings (4.2) are natural in the sense that the diagram

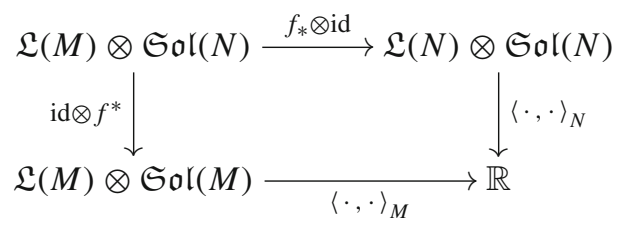

commutes, for all Loc-morphisms $f: M \rightarrow N$. Furthermore, one immediately observes that the chain maps $j$ in (4.4) are natural in the sense that the diagram

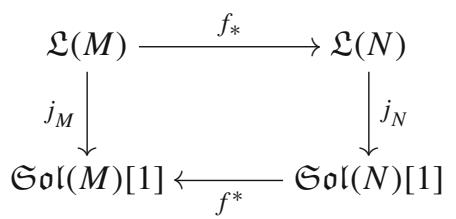

commutes, for all Loc-morphisms $f: M \rightarrow N$, and that the shifted Poisson structures $\Upsilon$ in (4.5) are natural in the sense that the diagram

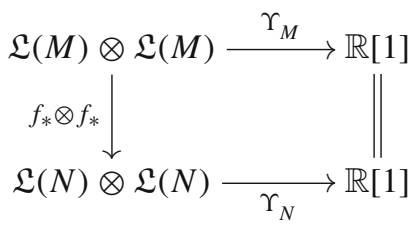

commutes, for all Loc-morphisms $f: M \rightarrow N$.

Remark 6.3. Note that all our assumptions above on naturality of vector bundles and differential operators are satisfied for our examples of interest given by Klein-Gordon theory (see Examples 3.2 and 3.8) and linear Yang-Mills theory (see Examples 3.3 and 3.9). In these examples $f^{*}$ is simply given by pullback of differential forms.

Remark 6.4. Using as in Remark 6.2 the forgetful functor $\mathfrak{U}_{\bar{M}}:$ Loc $/ \bar{M} \rightarrow$ Loc, all functors and natural transformations on Loc that we introduced above can be restricted to the slice category Loc $/ \bar{M}$, for each $\bar{M} \in \mathbf{L o c}$. This restricted data is sufficient when one attempts to construct only a homotopy AQFT on a fixed $\bar{M} \in \mathbf{L o c}$, in contrast to a homotopy AQFT on Loc. 
Our approach to construct unshifted Poisson structures (Definition 4.8) in terms of (compatible pairs of) retarded/advanced trivializations (Definition 4.4) has to be supplemented by a suitable naturality axiom. Because the strength of our final result will depend on whether we work with Loc or a slice category Loc $/ \bar{M}$, for some $\bar{M} \in \mathbf{L o c}$, we shall state our definitions and results below for both cases.

Definition 6.5. Let $\mathbf{C}$ be either Loc or Loc $/ \bar{M}$, for any $\bar{M} \in$ Loc. A C-natural retarded/advanced trivialization is a family

$$
\Lambda^{ \pm}:=\left\{\Lambda_{M}^{ \pm} \in \underline{\operatorname{hom}}\left(\mathfrak{L}_{\mathrm{pc} / \mathrm{fc}}(M), \mathfrak{L}_{\mathrm{pc} / \mathrm{fc}}(M)\right)_{1}\right\}_{M \in \mathbf{C}}
$$

of retarded/advanced trivializations for each $M \in \mathbf{C}$, such that

$$
f^{*}\left(j_{\mathrm{pc} / \mathrm{fc}} \Lambda_{N}^{ \pm} \iota\right) f_{*}=\left(j_{\mathrm{pc} / \mathrm{fc}} \Lambda_{M}^{ \pm} \iota\right)
$$

for all C-morphisms $f: M \rightarrow N$, see also (4.7).

Remark 6.6. Using as in Remark 6.2 the forgetful functor $\mathfrak{U}_{\bar{M}}:$ Loc $/ \bar{M} \rightarrow$ Loc, any Loc-natural retarded/advanced trivialization may be restricted to a Loc $/ \bar{M}$-natural retarded/advanced trivialization, for each $\bar{M} \in$ Loc. Thus, it is in general harder to construct Loc-natural retarded/advanced trivializations than Loc $/ \bar{M}$-natural ones.

Proposition 6.7. (a) The unique retarded/advanced trivializations for Klein-Gordon theory given in Proposition 4.12 define a Loc-natural retarded/advanced trivialization.

(b) The retarded/advanced trivializations for linear Yang-Mills theory given in Proposition 4.13 define a Loc-natural retarded/advanced trivialization.

Proof. This is an immediate consequence of the standard result that the retarded/advanced Green's operators $G_{M}^{ \pm}: \Omega_{\mathrm{c}}^{p}(M) \rightarrow \Omega^{p}(M)$ for the d'Alembert operator $\square$ or the Klein-Gordon operator $\square-m^{2}$ satisfy the naturality condition $f^{*} G_{N}^{ \pm} f_{*}=G_{M}^{ \pm}$, for all Loc-morphisms $f: M \rightarrow N$. See [BG11, Lemma 3.2] for a proof.

Definition 6.8. Let $\mathbf{C}$ be either Loc or Loc $/ \bar{M}$, for any $\bar{M} \in$ Loc. A C-natural unshifted Poisson structure on the solution complex functor $\mathfrak{S o l}: \mathbf{C}^{\text {op }} \rightarrow \mathbf{C h}_{\mathbb{R}}$ is a 0-cycle $\tau \in \underline{\operatorname{hom}}\left(\bigwedge^{2} \mathfrak{L}, \mathbb{R}\right)_{0}$ in the chain complex

$$
\underline{\operatorname{hom}}\left(\bigwedge^{2} \mathfrak{L}, \mathbb{R}\right):=\lim _{M \in \mathbf{C o p}} \underline{\operatorname{hom}}\left(\bigwedge^{2} \mathfrak{L}(M), \mathbb{R}\right) \in \mathbf{C h}_{\mathbb{R}},
$$

where $\mathfrak{L}: \mathbf{C} \rightarrow \mathbf{C h}_{\mathbb{R}}$ is the functor assigning chain complexes of linear observables. A C-natural homotopy between two $\mathbf{C}$-natural unshifted Poisson structures $\tau, \tilde{\tau} \in \underline{\operatorname{hom}}\left(\bigwedge^{2} \mathfrak{L}, \mathbb{R}\right)_{0}$ is a 1-chain $\rho \in \underline{\operatorname{hom}}\left(\bigwedge^{2} \mathfrak{L}, \mathbb{R}\right)_{1}$, such that $\tilde{\tau}-\tau=\partial \rho$.

Remark 6.9. We decided to state Definition 6.8 in a rather abstract form because this will become useful later. From a more concrete perspective, the data of a $\mathbf{C}$-natural unshifted Poisson structure $\tau \in \underline{\operatorname{hom}}\left(\bigwedge^{2} \mathfrak{L}, \mathbb{R}\right)_{0}$ is given by a family

$$
\left\{\tau_{M}: \mathfrak{L}(M) \wedge \mathfrak{L}(M) \rightarrow \mathbb{R}\right\}_{M \in \mathbf{C}}
$$

of chain maps, i.e. unshifted Poisson structures for each $M \in \mathbf{C}$, that satisfies the naturality condition

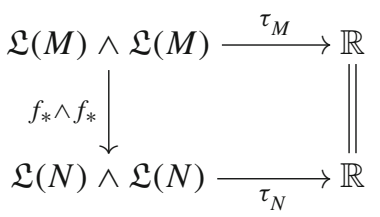


for all C-morphisms $f: M \rightarrow N$. Similarly, a $\mathbf{C}$-natural homotopy between $\tau$ and $\tilde{\tau}$ is a family $\left\{\rho_{M} \in \underline{\operatorname{hom}}\left(\bigwedge^{2} \mathfrak{L}(M), \mathbb{R}\right)_{1}\right\}_{M \in \mathbf{C}}$ of 1-chains, such that $\tilde{\tau}_{M}-\tau_{M}=\partial \rho_{M}$, for all $M \in \mathbf{C}$, and $\rho_{N}\left(f_{*} \wedge f_{*}\right)=\rho_{M}$, for all $\mathbf{C}$-morphisms $f: M \rightarrow N$. Similarly to Remark 6.6, we note that Loc-natural unshifted Poisson structures and their homotopies are harder to construct than Loc $/ \bar{M}$-natural ones.

Lemma 6.10. (a) Let $\mathbf{C}$ be either Loc or Loc $/ \bar{M}$, for any $\bar{M} \in \mathbf{L o c}$, and let $\Lambda^{ \pm}$be a C-natural compatible pair of retarded/advanced trivializations. Then the componentwise construction in Definition 4.8 defines a $\mathbf{C}$-natural unshifted Poisson structure $\tau$.

(b) Let $\mathbf{C}=\mathbf{L o c} / \bar{M}$, for any $\bar{M} \in$ Loc. Then the chain complex (6.10) is isomorphic to the mapping complex $\operatorname{hom}\left(\bigwedge^{2} \mathfrak{L}(\bar{M}), \mathbb{R}\right)$ corresponding to $\bar{M}$. As a consequence, every Loc $/ \bar{M}$-natural unshifted Poisson structure $\tau$ is uniquely determined by an unshifted Poisson structure $\tau_{\bar{M}}$ on $\bar{M}$ and every Loc $/ \bar{M}$-natural homotopy $\rho$ is uniquely determined by a homotopy $\rho_{\bar{M}}$ on $\bar{M}$.

(c) Suppose that $\Lambda^{ \pm}$and $\widetilde{\Lambda}^{ \pm}$are two Loc $/ \bar{M}$-natural compatible pairs of retarded/ advanced trivializations, for any $\bar{M} \in$ Loc. Then the corresponding Loc $/ \bar{M}$-natural unshifted Poisson structures $\tau, \tilde{\tau}$ from item (a) are homotopic, i.e. $\tilde{\tau}-\tau=\partial \rho$ for some Loc $/ \bar{M}$-natural homotopy $\rho$.

Proof. Item (a) is immediate because the definition of the unshifted Poisson structure in (4.10) involves only natural maps. Item (c) follows from item (b) and Corollary 4.10. It thus remains to prove item (b), which follows immediately from the fact that the slice category Loc $/ \bar{M}$ has a terminal object (id : $\bar{M} \rightarrow \bar{M}$ ), hence $(\text { Loc } / \bar{M})^{\text {op }}$ has an initial object. The limit in (6.10) is then isomorphic to the chain complex $\underline{\operatorname{hom}}\left(\bigwedge^{2} \mathfrak{L}(\bar{M}), \mathbb{R}\right)$ corresponding to this object.

Remark 6.11. It is currently unclear to us if the analog of Lemma 6.10 (c) also holds true for the category Loc. Let us explain this issue in more detail. Suppose that $\Lambda^{ \pm}, \widetilde{\Lambda}^{ \pm}$ are two Loc-natural compatible pairs of retarded/advanced trivializations and denote the corresponding Loc-natural unshifted Poisson structures by $\tau, \widetilde{\tau}$. By Corollary 4.10, we obtain that for every $M \in \mathbf{L o c}$ there exists a 1-chain $\rho_{M} \in \underline{\operatorname{hom}}\left(\bigwedge^{2} \mathfrak{L}(M), \mathbb{R}\right)_{1}$ such that $\tilde{\tau}_{M}-\tau_{M}=\partial \rho_{M}$. However, it is unclear whether such homotopies can be chosen to be Loc-natural as Loc has no terminal object. (The terminal object in Loc $/ \bar{M}$ was crucial to prove Lemma 6.10 (b) and hence (c).) As a consequence, it is currently unclear to us if the particular model for linear quantum Yang-Mills theory that we will construct below is, up to natural weak equivalences, the only possibility within our approach. In particular, we can not exclude the existence of a Loc-natural compatible pair of retarded/advanced trivializations different from the one in Proposition 6.7 (b), that leads to a non-homotopic Loc-natural unshifted Poisson structure and hence potentially to a non-equivalent quantization.

Example 6.12. Let us apply our general results to Klein-Gordon theory, see Examples 3.2 and 3.8 as well as Sect. 4.1. The Loc-natural compatible pair of retarded/advanced trivializations from Proposition 6.7 (a) defines via Lemma 6.10 (a) a Loc-natural unshifted Poisson structure $\tau^{\mathrm{KG}}$, whose components $\tau_{M}^{\mathrm{KG}}$, for $M \in \mathbf{L o c}$, are given concretely by (4.18). Due to the component-wise uniqueness result for retarded/advanced trivializations for Klein-Gordon theory in Proposition 4.12, it follows that $\tau^{\mathrm{KG}}$ is unique too. Hence, in the case of Klein-Gordon theory we obtain stronger results than in the general Lemma 6.10. 
Example 6.13. Let us now apply our general results to linear Yang-Mills theory, see Examples 3.3 and 3.9 as well as Sect. 4.2. The Loc-natural compatible pair of retarded/advanced trivializations from Proposition 6.7 (b) defines via Lemma 6.10 (a) a Loc-natural unshifted Poisson structure $\tau^{\mathrm{YM}}$, whose components $\tau_{M}^{\mathrm{YM}}$, for $M \in \mathbf{L o c}$, are given concretely by (4.26). Unfortunately, as explained in Remark 6.11, we are currently unable to exclude the existence of other Loc-natural choices of compatible pairs of retarded/advanced trivializations that define non-homotopic Loc-natural unshifted Poisson structures. The situation gets much better when we work on a slice category Loc $/ \bar{M}$, for any $\bar{M} \in \mathbf{L o c}$. In this case Proposition 6.7 (b) restricts to a Loc $/ \bar{M}$ natural compatible pair of retarded/advanced trivializations and Lemma 6.10 (a) defines a Loc $/ \bar{M}$-natural unshifted Poisson structure $\tau^{\mathrm{YM}}$. By Lemma 6.10 (c), we know that any other choice of a Loc $/ \bar{M}$-natural compatible pair of retarded/advanced trivializations defines a homotopic Loc $/ \bar{M}$-natural unshifted Poisson structure. This means that, when restricted to $\mathbf{L o c} / \bar{M}$, our constructions determine uniquely a homology class $\left[\tau^{\mathrm{YM}}\right]$ in $H_{0}\left(\underline{\operatorname{hom}}\left(\bigwedge^{2} \mathfrak{L}^{\mathrm{YM}}, \mathbb{R}\right)\right)$.

Let $\mathbf{C}$ be either Loc or Loc $/ \bar{M}$, for any $\bar{M} \in \mathbf{L o c}$, and suppose that we picked a C-natural unshifted Poisson structure $\tau$. The assignment $M \mapsto\left(\mathfrak{L}(M), \tau_{M}\right)$ defines a functor

$$
(\mathfrak{L}, \tau): \mathbf{C} \longrightarrow \mathbf{P o C h}_{\mathbb{R}}
$$

to the category of unshifted Poisson complexes, and post-composition with the CCR quantization functor (5.4) defines a functor

$$
\mathfrak{A}:=\mathfrak{C} \mathfrak{C}(\mathfrak{L}, \tau): \mathbf{C} \longrightarrow \mathbf{d g}^{*} \mathbf{A l g} \mathbf{C}_{\mathbb{C}}
$$

to the category of differential graded $*$-algebras.

In order to analyze homotopical properties of this construction, we endow both functor categories $\operatorname{Fun}\left(\mathbf{C}, \operatorname{PoCh}_{\mathbb{R}}\right)$ and $\operatorname{Fun}\left(\mathbf{C}, \mathbf{d g}^{*} \mathbf{A} \mathbf{I g}_{\mathbb{C}}\right)$ with the structure of a homotopical category [DHKS04, Rie14] in which weak equivalences are so-called natural weak equivalences.

Definition 6.14. Let $\mathbf{C}$ be either Loc or Loc $/ \bar{M}$, for any $\bar{M} \in \mathbf{L o c}$.

(i) A morphism in $\mathbf{F u n}\left(\mathbf{C}, \mathbf{P o C h}_{\mathbb{R}}\right)$ (i.e. a natural transformation) is a natural weak equivalence if all its components are weak equivalences in $\mathbf{P o C h} \mathbf{h}_{\mathbb{R}}$, see Definition 5.2.

(ii) A morphism in $\mathbf{F u n}\left(\mathbf{C}, \mathbf{d g}^{*} \mathbf{A l g} \mathbf{g}_{\mathbb{C}}\right.$ ) (i.e. a natural transformation) is a natural weak equivalence if all its components are weak equivalences in $\mathbf{d g}^{*} \mathbf{A} \mathbf{I g}_{\mathbb{C}}$, see Definition 5.2.

(iii) Let $\mathbf{h A Q F T}(\mathbf{C}) \subseteq \operatorname{Fun}\left(\mathbf{C}, \mathbf{d g}^{*} \mathbf{A l g} \mathbf{C}_{\mathbb{C}}\right)$ denote the full subcategory of functors satisfying the homotopy AQFT axioms from Definition 6.1. A morphism in $\operatorname{hAQFT}(\mathbf{C})$ is a weak equivalence if and only if it is a natural weak equivalence in $\operatorname{Fun}\left(\mathbf{C}, \operatorname{dg}^{*} \operatorname{Alg}_{\mathbb{C}}\right)$.

Remark 6.15. Note that the weak equivalences in $\mathbf{h A Q F T}(\mathbf{C})$ agree with those considered in [BSW19b].

The following result generalizes Proposition 5.3 to the context of functor categories.

Proposition 6.16. Let $\mathbf{C}$ be either Loc or Loc $/ \bar{M}$, for any $\bar{M} \in$ Loc. 
(a) Post-composition with the CCR functor defines a homotopical functor

$$
\mathfrak{C C} \cap(-): \operatorname{Fun}\left(C, \operatorname{PoCh}_{\mathbb{R}}\right) \longrightarrow \operatorname{Fun}\left(C, \operatorname{dg}^{*} \operatorname{Alg}_{\mathbb{C}}\right) \text {. }
$$

(b) Let $(V, \tau) \in \operatorname{Fun}\left(\mathbf{C}, \operatorname{PoCh} \mathbf{h}_{\mathbb{R}}\right)$ and $\rho \in \underline{\operatorname{hom}}\left(\bigwedge^{2} V, \mathbb{R}\right)_{1}$ a $\mathbf{C}$-natural 1-chain. Then there exists a zig-zag of natural weak equivalences in $\mathbf{F u n}\left(\mathbf{C}, \mathbf{d g}^{*} \mathbf{A l g} \mathbf{g}_{\mathbb{C}}\right)$ connecting $\mathfrak{C C} \mathfrak{R}(V, \tau)$ and $\mathfrak{C} \mathfrak{R}(V, \tau+\partial \rho)$.

Proof. Item (a) is an immediate consequence of the component-wise definition of natural weak equivalences in Definition 6.14 and the result in Proposition 5.3 (a) that the CCR functor is a homotopical functor.

Let us now focus on item (b). By Proposition 5.3 (b) and the explicit construction in Proposition A.3, we obtain for each object $M \in \mathbf{C}$ a zig-zag

$$
\mathfrak{C C} \Re\left(V(M), \tau_{M}\right) \stackrel{\sim}{\longleftarrow} \mathfrak{Q}_{\operatorname{lin}}\left(H_{\left(V(M), \tau_{M}, \rho_{M}\right)} \stackrel{\sim}{\longrightarrow} \mathfrak{C C} \mathfrak{R}\left(V(M), \tau_{M}+\partial \rho_{M}\right)\right.
$$

of weak equivalences in $\mathbf{d g}^{*} \mathbf{A} \mathbf{l} \mathbf{g}_{\mathbb{C}}$. From our construction of the object $H_{\left(V(M), \tau_{M}, \rho_{M}\right)}$ in Proposition A.3, one immediately observes that (6.15) are the components of a zig-zag of natural weak equivalences.

Together with Lemma 6.10 (c), Proposition 6.16 (b) implies the following important result.

Corollary 6.17. Fix any $\bar{M} \in$ Loc and suppose that $\Lambda^{ \pm}$and $\widetilde{\Lambda}^{ \pm}$are two Loc $/ \bar{M}$ natural compatible pairs of retarded/advanced trivializations. Denote the corresponding Loc $/ \bar{M}$-natural unshifted Poisson structures from Lemma 6.10 (a) by $\tau$ and $\tilde{\tau}$. Then the two functors $\mathfrak{A}:=\mathfrak{C} \mathfrak{C}(\mathfrak{L}, \tau)$ and $\widetilde{\mathfrak{A}}:=\mathfrak{C} \mathfrak{C}(\mathfrak{L}, \widetilde{\tau})$ are equivalent via a zig-zag of natural weak equivalences in $\mathbf{F u n}\left(\mathbf{L o c} / \bar{M}, \mathbf{d g}^{*} \mathbf{A l g} \mathbf{C}_{\mathbb{C}}\right)$.

The next lemma provides conditions on $(\mathfrak{L}, \tau): \mathbf{C} \rightarrow \mathbf{P o C h}_{\mathbb{R}}$ which imply that $\mathfrak{A}:=\mathfrak{C} \mathfrak{C}(\mathfrak{L}, \tau): \mathbf{C} \rightarrow \mathbf{d g}^{*} \mathbf{A l g}_{\mathbb{C}}$ fulfills the homotopy AQFT axioms from Definition 6.1 .

Lemma 6.18. Let $\mathbf{C}$ be either Loc or Loc $/ \bar{M}$, for any $\bar{M} \in \mathbf{L o c}$, and consider a functor $(\mathfrak{L}, \tau): \mathbf{C} \rightarrow \mathbf{P o C h}_{\mathbb{R}}$.

(a) If for every pair $\left(f_{1}: M_{1} \rightarrow N, f_{2}: M_{2} \rightarrow N\right)$ of $\mathbf{C}$-morphisms with causally disjoint images the chain map

$$
\tau\left(f_{1 *} \otimes f_{2 *}\right): \mathfrak{L}\left(M_{1}\right) \otimes \mathfrak{L}\left(M_{2}\right) \longrightarrow \mathfrak{L}(N)
$$

is zero, then the functor $\mathfrak{A}:=\mathfrak{C} \mathfrak{C}(\mathfrak{L}, \tau): \mathbf{C} \rightarrow \mathbf{d g}^{*} \mathbf{A} \mathbf{l g}_{\mathbb{C}}$ satisfies Einstein causality.

(b) If for every Cauchy morphism $f: M \rightarrow N$ the chain map $f_{*}: \mathfrak{L}(M) \rightarrow \mathfrak{L}(N)$ is a quasi-isomorphism, then the functor $\mathfrak{A}:=\mathfrak{C} \mathfrak{C}(\mathfrak{L}, \tau): \mathbf{C} \rightarrow \mathbf{d g}^{*} \mathbf{A l g}_{\mathbb{C}}$ satisfies time-slice.

Proof. Item (a) is a direct consequence of the canonical commutation relations in (5.3). Item (b) follows from the fact that $\mathfrak{C} \mathfrak{R}$ is a homotopical functor, see Proposition 5.3 (a).

We are now ready to state and prove the main result of the present paper. 
Theorem 6.19. (a) Let $\tau^{\mathrm{KG}}$ denote the unshifted Poisson structure defined by Lemma 6.10 (a) from the unique Loc-natural compatible pair of retarded/advanced trivializations for Klein-Gordon theory, see Proposition 6.7 (a). Then the functor $\mathfrak{A}^{\mathrm{KG}}:=\mathfrak{C} \mathfrak{C}\left(\mathfrak{L}^{\mathrm{KG}}, \tau^{\mathrm{KG}}\right):$ Loc $\rightarrow \mathbf{d g}^{*} \mathbf{A l g} \mathbb{C}$ is a homotopy AQFT on Loc, i.e. $\mathfrak{A}^{\mathrm{KG}} \in \mathbf{h A Q F T}(\mathbf{L o c})$.

(b) Let $\tau^{\mathrm{YM}}$ denote the unshifted Poisson structure defined by Lemma 6.10 (a) from the Loc-natural compatible pair of retarded/advanced trivializations for linear YangMills theory, see Proposition 6.7 (b). Then the functor $\mathfrak{A}^{\mathrm{YM}}:=\mathfrak{C} \mathfrak{C}\left(\mathfrak{L}^{\mathrm{YM}}, \tau^{\mathrm{YM}}\right)$ : Loc $\rightarrow \mathbf{d g}^{*} \mathbf{A l g} \mathbb{C}_{\mathbb{C}}$ is a homotopy AQFT on Loc, i.e. $\mathfrak{A}^{\mathrm{YM}} \in \mathbf{h A Q F T}(\mathbf{L o c})$. The restriction $\mathfrak{A} \frac{Y M}{M}:=\mathfrak{A}^{\mathrm{YM}} \mathfrak{U}_{\bar{M}} \in \mathbf{h A Q F T}(\mathbf{L o c} / \bar{M})$ given in Remark 6.2 defines a homotopy AQFT on each $\bar{M} \in$ Loc. Up to natural weak equivalence, these homotopy AQFTs on $\bar{M}$ do not depend on the choice of a Loc-natural compatible pair of retarded/advanced trivializations.

Proof. Item (a): Example 6.12 defines a functor $\left(\mathfrak{L}^{\mathrm{KG}}, \tau^{\mathrm{KG}}\right): \mathbf{L o c} \rightarrow \mathbf{P o C h}_{\mathbb{R}}$ and hence by post-composition with $\mathfrak{C} \mathfrak{C}$ a functor $\mathfrak{A}^{\mathrm{KG}}:=\mathfrak{C} \mathfrak{C} \mathfrak{R}\left(\mathfrak{L}^{\mathrm{KG}}, \tau^{\mathrm{KG}}\right):$ Loc $\rightarrow$ $\mathbf{d g}^{*} \mathbf{A} \mathbf{I g}_{\mathbb{C}}$. It remains to prove that this functor satisfies the homotopy AQFT axioms from Definition 6.1, which we shall do by checking the sufficient conditions on $\left(\mathfrak{L}^{\mathrm{KG}}, \tau^{\mathrm{KG}}\right)$ from Lemma 6.18. We deduce from the explicit expressions for $\tau^{\mathrm{KG}}$ in (4.18) and the support properties of retarded/advanced Green's operators (see Sect. 2.1) that the hypothesis of Lemma 6.18 (a) is fulfilled, hence $\mathfrak{A}^{\mathrm{KG}}$ satisfies Einstein causality.

In order to prove time-slice, recall from Example 5.5 that the quotient maps $\left(\mathfrak{L}^{\mathrm{KG}}(M), \tau_{M}^{\mathrm{KG}}\right) \rightarrow\left(H_{0}\left(\mathfrak{L}^{\mathrm{KG}}(M)\right), \tau_{M}^{\mathrm{KG}}\right)$ are weak equivalences in $\mathbf{P o C h} \mathbf{C h}_{\mathbb{R}}$ for every $M \in$ Loc. This clearly defines a natural weak equivalence $\left(\mathfrak{L}^{\mathrm{KG}}, \tau^{\mathrm{KG}}\right) \rightarrow$ $\left(H_{0}\left(\mathfrak{L}^{\mathrm{KG}}\right), \tau^{\mathrm{KG}}\right)$ in $\mathbf{F u n}\left(\mathbf{L o c}, \mathbf{P o C h} \mathbf{C}_{\mathbb{R}}\right)$, hence we may equivalently prove that $\left(H_{0}\left(\mathfrak{L}^{\mathrm{KG}}\right), \tau^{\mathrm{KG}}\right)$ fulfills the hypothesis of Lemma $6.18\left(\right.$ b), i.e. $H_{0}\left(f_{*}\right): H_{0}\left(\mathfrak{L}^{\mathrm{KG}}(M)\right) \rightarrow$ $H_{0}\left(\mathfrak{L}^{\mathrm{KG}}(N)\right)$ is a quasi-isomorphism (i.e. an isomorphism because both chain complexes are concentrated in degree zero) for every Cauchy morphism $f: M \rightarrow N$. Because $H_{0}\left(\mathfrak{L}^{\mathrm{KG}}\right)$ describes the usual vector spaces of linear on-shell observables for Klein-Gordon theory, this follows from standard results in the literature, see e.g. [BD15, Theorem 3.3.1]. This shows that $\mathfrak{A}^{\mathrm{KG}}: \mathbf{L o c} \rightarrow \mathbf{d g}^{*} \mathbf{A} \mathbf{l g}_{\mathbb{C}}$ satisfies the homotopy AQFT axioms, hence it is a homotopy AQFT on Loc.

Item $(b)$ : Example 6.13 defines a functor $\left(\mathfrak{L}^{\mathrm{YM}}, \tau^{\mathrm{YM}}\right):$ Loc $\rightarrow \mathbf{P o C h}_{\mathbb{R}}$ and hence by post-composition with $\mathfrak{C} \mathfrak{R}$ a functor $\mathfrak{A}^{\mathrm{YM}}:=\mathfrak{C} \mathfrak{C} \mathfrak{R}\left(\mathfrak{L}^{\mathrm{YM}}, \tau^{\mathrm{YM}}\right): \mathbf{L o c} \rightarrow \mathbf{d g}^{*} \mathbf{A} \mathbf{I g}_{\mathbb{C}}$. We prove that this functor satisfies the homotopy AQFT axioms from Definition 6.1 by checking the sufficient conditions on $\left(\mathfrak{L}^{\mathrm{YM}}, \tau^{\mathrm{YM}}\right)$ from Lemma 6.18. We deduce from the explicit expressions for $\tau^{\mathrm{YM}}$ in (4.26) and the support properties of retarded/advanced Green's operators (see Sect. 2.1) that the hypothesis of Lemma 6.18 (a) is fulfilled, hence $\mathfrak{A}^{\mathrm{YM}}$ satisfies Einstein causality.

Our next aim is to prove that the hypothesis of Lemma 6.18 (b) is fulfilled too, which would imply that $\mathfrak{A}^{\mathrm{YM}}$ satisfies time-slice. Let $f: M \rightarrow N$ be any Cauchy morphism and consider the chain map $f_{*}: \mathfrak{L}^{\mathrm{YM}}(M) \rightarrow \mathfrak{L}^{\mathrm{YM}}(N)$, where $\mathfrak{L}^{\mathrm{YM}}$ is concretely given in (4.19). We have to prove that the induced map $H_{n}\left(f_{*}\right): H_{n}\left(\mathfrak{L}^{\mathrm{YM}}(M)\right) \rightarrow H_{n}\left(\mathfrak{L}^{\mathrm{YM}}(N)\right)$ in homology is an isomorphism, for every $n \in \mathbb{Z}$. From the explicit computation of homologies performed in Sect. 4.2, we find that the only non-trivial homologies are in degrees $n=-1,0,1$, hence we can restrict our attention to these cases. The homologies in degrees $n= \pm 1$ are compactly supported de Rham cohomologies, hence $H_{n}\left(f_{*}\right)$ is an isomorphism in these degrees because of Poincare duality, homotopy invariance of de Rham cohomology and the fact that every Cauchy morphism $f: M \rightarrow N$ is in particular a homotopy equivalence. In degree $n=0$, the linear map $H_{0}\left(f_{*}\right)$ : 
$H_{0}\left(\mathfrak{L}^{\mathrm{YM}}(M)\right) \rightarrow H_{0}\left(\mathfrak{L}^{\mathrm{YM}}(N)\right)$ is the usual push-forward along $f$ of linear gaugeinvariant on-shell observables for linear Yang-Mills theory, which is known to be an isomorphism, see e.g. [SDH14,BDS14,BDHS14,FL16, Ben16, BSS16].

Summing up, this shows that $\mathfrak{A}^{\mathrm{YM}}: \mathbf{L o c} \rightarrow \mathbf{d g}^{*} \mathbf{A l g} \mathbb{C}_{\mathbb{C}}$ satisfies the homotopy AQFT axioms, hence it is a homotopy AQFT on Loc. The statement about uniqueness (up to natural weak equivalences) for the restricted linear Yang-Mills homotopy AQFTs $\mathfrak{A} \frac{\mathrm{YM}}{M} \in \mathbf{h A Q F T}(\mathbf{L o c} / \bar{M})$, for each $\bar{M} \in \mathbf{L o c}$, is a consequence of Corollary 6.17.

Remark 6.20. Note that our particular model in Theorem 6.19 (a) for Klein-Gordon theory as a homotopy AQFT on Loc is given by a functor $\mathfrak{A}^{\mathrm{KG}}$ that assigns a differential graded $*$-algebra to each $M \in$ Loc. This is seemingly different to the usual description of Klein-Gordon theory as a functor with values in ordinary *algebras, see e.g. [BDH13]. These two descriptions are however equivalent via a natural weak equivalence in the homotopical category hAQFT(Loc). Concretely, in the proof of Theorem 6.19 (a) we observed that there exists a natural weak equivalence $\left(\mathfrak{L}^{\mathrm{KG}}, \tau^{\mathrm{KG}}\right) \rightarrow\left(H_{0}\left(\mathfrak{L}^{\mathrm{KG}}\right), \tau^{\mathrm{KG}}\right)$ between our unshifted Poisson complexes for KleinGordon theory and their zeroth homologies, which are the structures of interest in the usual description of Klein-Gordon theory. Proposition 6.16 (a) then implies that $\mathfrak{A}^{\mathrm{KG}}=\mathfrak{C} \mathfrak{C} \mathfrak{R}\left(\mathfrak{L}^{\mathrm{KG}}, \tau^{\mathrm{KG}}\right) \simeq \mathfrak{C} \mathfrak{C}\left(H_{0}\left(\mathfrak{L}^{\mathrm{KG}}\right), \tau^{\mathrm{KG}}\right)$ is a natural weak equivalence in hAQFT(Loc), i.e. our description of Klein-Gordon theory as a homotopy AQFT is equivalent to the usual one in e.g. [BDH13].

Remark 6.21. As we have already indicated in Remark 6.11 and Example 6.13, at the moment we cannot exclude the possibility that there exists another Loc-natural compatible pair of advanced/retarded trivializations for linear Yang-Mills theory that defines a non-homotopic Loc-natural unshifted Poisson structure, and hence a potentially nonequivalent homotopy AQFT $\widetilde{\mathfrak{A}}^{\mathrm{YM}} \in \mathbf{h A Q F T}($ Loc $)$. Note that potential differences would be very subtle because, as a consequence of Theorem 6.19 (b), the restrictions $\widetilde{\mathfrak{A}} \bar{M}, \mathfrak{A}_{\bar{M}}^{\mathrm{YM}} \in \mathbf{h A Q F T}(\mathbf{L o c} / \bar{M})$ to every $\bar{M} \in$ Loc are naturally weakly equivalent homotopy AQFTs on $\bar{M}$.

In contrast to the situation for Klein-Gordon theory explained in Remark 6.20, our model in Theorem 6.19 (b) for linear Yang-Mills theory as a homotopy AQFT on Loc is not naturally weakly equivalent to existing models in the literature that consider only gauge-invariant on-shell observables, see e.g. [SDH14,BDS14, BDHS14, FL16, Ben16, BSS16]. This is because, on a generic $M \in$ Loc, the complex of linear observables $\mathfrak{L}^{\mathrm{YM}}(M)$ has non-trivial homology in degrees $n=-1,0,1$, while the usual models in the literature consider only its zeroth homology. In the terminology of the BRST/BV formalism, one can say that our description of linear Yang-Mills theory as a homotopy AQFT $\mathfrak{A}^{\mathrm{YM}} \in \mathbf{h A Q F T}(\mathbf{L o c})$ takes fully into account all ghost and antifield observables, while the traditional models consider only the 0 -truncation of the antifield number 0 sector of the theory. In particular, notice that the difference between $\mathfrak{A}^{\mathrm{YM}}(M)$ and $\mathfrak{C C} \mathfrak{R}\left(H_{0}\left(\mathfrak{L}^{\mathrm{YM}}(M)\right), \tau_{M}^{\mathrm{YM}}\right)$, for a generic $M \in \mathbf{L o c}$, is already visible on the level of the zeroth homology: The $*$-algebra $\mathfrak{C} \mathfrak{C} \mathfrak{R}\left(H_{0}\left(\mathfrak{L}^{\mathrm{YM}}(M)\right), \tau_{M}^{\mathrm{YM}}\right)$ is generated only by linear gauge-invariant on-shell observables, while the $*$-algebra $H_{0}\left(\mathfrak{A}^{\mathrm{YM}}(M)\right)$ contains also classes that are obtained by multiplying in $\mathfrak{A}^{\mathrm{YM}}(M)$ an equal number of ghost field and antifield linear observables.

Acknowledgements. We would like to thank the anonymous referees for valuable comments that helped us to improve this manuscript. We also would like to thank Chris Fewster, Owen Gwilliam, Igor Khavkine, François Petit and Nic Teh for useful discussions and comments on this work. The work of M.B. was supported by 
a Research Grant funded by the Deutsche Forschungsgemeinschaft (DFG, Germany). S.B. is supported by a PhD scholarship of the Royal Society (UK). A.S. gratefully acknowledges the financial support of the Royal Society (UK) through a Royal Society University Research Fellowship, a Research Grant and an Enhancement Award.

Open Access This article is distributed under the terms of the Creative Commons Attribution 4.0 International License (http://creativecommons.org/licenses/by/4.0/), which permits unrestricted use, distribution, and reproduction in any medium, provided you give appropriate credit to the original author(s) and the source, provide a link to the Creative Commons license, and indicate if changes were made.

Publisher's Note Springer Nature remains neutral with regard to jurisdictional claims in published maps and institutional affiliations.

\section{A. Technical Details for Proposition 5.3}

In this technical appendix we shall use quite freely the techniques and results developed in [BS18]. Let us first recall from [BS18] that the CCR functor (5.4) admits a factorization

$$
\operatorname{PoCh}_{\mathbb{R}} \underset{\mathfrak{H e i S}^{\prime}}{\longrightarrow} \text { dg }^{*} \mathbf{u L i e}_{\mathbb{C}} \underset{\mathfrak{Q}_{\operatorname{lin}}}{\longrightarrow} \text { dg }^{*} \mathbf{A l g}_{\mathbb{C}}
$$

through the homotopical category of differential graded unital Lie *-algebras, where $\mathfrak{H e i s}: \mathbf{P o C h}_{\mathbb{R}} \rightarrow \mathbf{d g}^{*} \mathbf{u L i e}_{\mathbb{C}}$ is the Heisenberg Lie algebra functor and $\mathfrak{Q}_{\text {lin }}$ : $\mathbf{d g}^{*} \mathbf{u L i e} \mathbb{C} \rightarrow \mathbf{d g}^{*} \mathbf{A} \mathbf{I g}_{\mathbb{C}}$ is the unital universal enveloping algebra functor. Our strategy for proving Proposition 5.3 is to prove the analogous statements for the Heisenberg Lie algebra functor $\mathfrak{H e i s}: \mathbf{P o C h}_{\mathbb{R}} \rightarrow \mathbf{d g}^{*} \mathbf{u L i e} \mathbb{C}_{\mathbb{C}}$. This will imply our desired results, because of the following

Lemma A.1 ([BS18]). $\mathfrak{Q}_{\text {lin }}: \mathbf{d g}^{*} \mathbf{u L i e} \mathbb{C}_{\mathbb{C}} \rightarrow \mathbf{d g}^{*} \mathbf{A} \mathbf{l g}_{\mathbb{C}}$ is a homotopical functor.

Let us recall that the Heisenberg Lie algebra $\mathfrak{H e i s}(V, \tau) \in \mathbf{d g}^{*} \mathbf{u L i e} \mathbb{C}_{\mathbb{C}}$ associated to an unshifted Poisson complex $(V, \tau) \in \mathbf{P o C h}_{\mathbb{R}}$ is given by the chain complex

$$
\mathfrak{H e i s}(V, \tau):=V_{\mathbb{C}} \oplus \mathbb{C}
$$

together with the Lie bracket $[-,-]:\left(V_{\mathbb{C}} \oplus \mathbb{C}\right) \otimes\left(V_{\mathbb{C}} \oplus \mathbb{C}\right) \rightarrow V_{\mathbb{C}} \oplus \mathbb{C}$ determined by

$$
\left[v_{1} \oplus c_{1}, v_{2} \oplus c_{2}\right]=0 \oplus i \tau\left(v_{1}, v_{2}\right),
$$

for all $v_{1}, v_{2} \in V$ and $c_{1}, c_{2} \in \mathbb{C}$, and unit $\eta: \mathbb{C} \rightarrow V_{\mathbb{C}} \oplus \mathbb{C}$ given by $\mathbb{1}:=\eta(1)=0 \oplus 1$. The $*$-involution on $V_{\mathbb{C}} \oplus \mathbb{C}$ is determined by $v^{*}=v$, for all $v \in V$, and complex conjugation on $\mathbb{C}$. To a morphism $f:(V, \tau) \rightarrow\left(V^{\prime}, \tau^{\prime}\right)$ in $\mathbf{P o C h}_{\mathbb{R}}$ it assigns the $\operatorname{dg}^{*} \mathbf{u L i e}_{\mathbb{C}}$-morphism $\mathfrak{H e i s}(f): \mathfrak{H e i s}(V, \tau) \rightarrow \mathfrak{H e i s}\left(V^{\prime}, \tau^{\prime}\right)$ determined by $f_{\mathbb{C}} \oplus$ id : $V_{\mathbb{C}} \oplus \mathbb{C} \rightarrow V_{\mathbb{C}}^{\prime} \oplus \mathbb{C}$, where $f_{\mathbb{C}}:=f \otimes \mathrm{id}: V \otimes \mathbb{C} \rightarrow V^{\prime} \otimes \mathbb{C}$ denotes the complexification of the chain map $f$.

The following lemma follows directly from the definitions.

Lemma A.2. Heis : $\mathbf{P o C h}_{\mathbb{R}} \rightarrow$ dg*uLie $_{\mathbb{C}}$ is a homotopical functor. Together with Lemma A.1, this proves Proposition 5.3 (a).

The main technical result of this appendix is 
Proposition A.3. Let $(V, \tau) \in \mathbf{P o C h}_{\mathbb{R}}$ be an unshifted Poisson complex and $\rho \in$ $\underline{\operatorname{hom}}\left(\bigwedge^{2} V, \mathbb{R}\right)_{1}$ a 1-chain. Then there exists a zig-zag $\mathfrak{H e i s}(V, \tau) \stackrel{\sim}{\leftarrow} H_{(V, \tau, \rho)} \stackrel{\sim}{\rightarrow}$ $\overline{\mathfrak{H e i s}}(V, \tau+\partial \rho)$ of weak equivalences in $\mathbf{d g}^{*} \mathbf{u L i e} \mathbb{C}_{\mathbb{C}}$. Together with Lemma A.1, this proves Proposition 5.3 (b).

Proof. We construct an explicit object $H_{(V, \tau, \rho)} \in \mathbf{d g}^{*} \mathbf{u L i e}_{\mathbb{C}}$ that allows us to exhibit the desired zig-zag of weak equivalences. Let us introduce the acyclic chain complex

$$
D:=(\stackrel{(-1)}{\mathbb{C}} \stackrel{\text { id }}{\longleftarrow} \stackrel{(0)}{\mathbb{C}}) \in \mathbf{C h}_{\mathbb{C}}
$$

and the notations $x:=1 \in D_{0}$ and $y:=\mathrm{d} x=1 \in D_{-1}$. We define $H_{(V, \tau, \rho)} \in \mathbf{d g}^{*} \mathbf{u L i e} \mathbb{C}$ by

$$
H_{(V, \tau, \rho)}:=V_{\mathbb{C}} \oplus D \oplus \mathbb{C},
$$

together with the unit $\mathbb{1}:=0 \oplus 0 \oplus 1$ and the Lie bracket

$$
\left[v_{1} \oplus \alpha_{1} \oplus c_{1}, v_{2} \oplus \alpha_{2} \oplus c_{2}\right]:=0 \oplus\left(i \partial \rho\left(v_{1}, v_{2}\right) x+i \rho\left(v_{1}, v_{2}\right) y\right) \oplus i \tau\left(v_{1}, v_{2}\right) \text {. }
$$

For any real number $s \in \mathbb{R}$, we let $\mathcal{I}_{s} \subseteq H_{(V, \tau, \rho)}$ be the differential graded unital Lie *-algebra ideal generated by the two relations

$$
0 \oplus x \oplus 0=0 \oplus 0 \oplus s, \quad 0 \oplus y \oplus 0=0 .
$$

Note that the corresponding quotient

$$
H_{(V, \tau, \rho)} / \mathcal{I}_{S} \cong \mathfrak{H e i s}(V, \tau+s \partial \rho)
$$

is isomorphic to the Heisenberg Lie algebra of $(V, \tau+s \partial \rho) \in \mathbf{P o C h}_{\mathbb{R}}$. We still have to show that the quotient map

$$
\pi_{s}: H_{(V, \tau, \rho)} \longrightarrow \mathfrak{H e i s}(V, \tau+s \partial \rho)
$$

is a weak equivalence in $\mathbf{d g}^{*} \mathbf{u L i e} \mathbf{C}_{\mathbb{C}}$. From the explicit form of the relations in (A.7), we observe that $\pi_{s}=\operatorname{id}_{V} \oplus q_{s}$ with $q_{s}: D \oplus \mathbb{C} \rightarrow \mathbb{C}$ given by $q_{s}:\left(c_{1} x+c_{2} y\right) \oplus c_{3} \mapsto$ $s c_{1}+c_{3}$. This is clearly a quasi-isomorphism in $\mathbf{C h}_{\mathbb{C}}$, hence (A.9) is a weak equivalence for any $s \in \mathbb{R}$. The desired zig-zag follows by taking $s=0$ and $s=1$.

\section{References}

[Bar15] Bär, C.: Green-hyperbolic operators on globally hyperbolic spacetimes. Commun. Math. Phys. 333(3), 1585 (2015). arXiv:1310.0738 [math-ph]

[BGP07] Bär, C., Ginoux, N., Pfäffle, F.: Wave equations on Lorentzian manifolds and quantization. Eur. Math. Soc. Zürich (2007). arXiv:0806.1036 [math.DG]

[BG11] Bär, C., Ginoux, N.: Classical and quantum fields on Lorentzian manifolds. Springer Proc. Math. 17, 359 (2011). arXiv:1104.1158 [math-ph]

[BBSS17] Becker, C., Benini, M., Schenkel, A., Szabo, R.J.: Abelian duality on globally hyperbolic spacetimes. Commun. Math. Phys. 349(1), 361 (2017). arXiv:1511.00316 [hep-th]

[BSS16] Becker, C., Schenkel, A., Szabo, R.J.: Differential cohomology and locally covariant quantum field theory. Rev. Math. Phys. 29(01), 1750003 (2016). arXiv:1406.1514 [hep-th]

[Ben16] Benini, M.: Optimal space of linear classical observables for Maxwell $k$-forms via spacelike and timelike compact de Rham cohomologies. J. Math. Phys. 57(5), 053502 (2016). arXiv: 1401.7563 [math-ph] 
[BD15] Benini, M., Dappiaggi, C.: Models of free quantum field theories on curved backgrounds. In: Brunetti, R., Dappiaggi, C., Fredenhagen, K., Yngvason, J. (eds.) Advances in Algebraic Quantum Field Theory. Springer, Heidelberg (2015)

[BDH13] Benini, M., Dappiaggi, C., Hack, T.P.: Quantum field theory on curved backgrounds-a primer. Int. J. Mod. Phys. A 28, 1330023 (2013). arXiv:1306.0527 [gr-qc]

[BDHS14] Benini, M., Dappiaggi, C., Hack, T.P., Schenkel, A.: A $C^{*}$-algebra for quantized principal $U$ (1)-connections on globally hyperbolic Lorentzian manifolds. Commun. Math. Phys. 332, 477 (2014). arXiv:1307.3052 [math-ph]

[BDM14] Benini, M., Dappiaggi, C., Murro, S.: Radiative observables for linearized gravity on asymptotically flat spacetimes and their boundary induced states. J. Math. Phys. 55, 082301 (2014). arXiv: 1404.4551 [gr-qc]

[BDS14] Benini, M., Dappiaggi, C., Schenkel, A.: Quantized Abelian principal connections on Lorentzian manifolds. Commun. Math. Phys. 330, 123 (2014). arXiv:1303.2515 [math-ph]

[BS17] Benini, M., Schenkel, A.: Quantum field theories on categories fibered in groupoids. Commun. Math. Phys. 356(1), 19 (2017). arXiv:1610.06071 [math-ph]

[BS19] Benini, M., Schenkel, A.: Higher structures in algebraic quantum field theory. Fortschr. Phys. 67(8-9), 1910015 (2019). arXiv:1903.02878 [hep-th]

[BSS18] Benini, M., Schenkel, A., Schreiber, U.: The stack of Yang-Mills fields on Lorentzian manifolds. Commun. Math. Phys. 359(2), 765 (2018). arXiv:1704.01378 [math-ph]

[BSS15] Benini, M., Schenkel, A., Szabo, R.J.: Homotopy colimits and global observables in Abelian gauge theory. Lett. Math. Phys. 105(9), 1193 (2015). arXiv:1503.08839 [math-ph]

[BSW17] Benini, M., Schenkel, A., Woike, L.: Operads for algebraic quantum field theory (2017). arXiv:1709.08657 [math-ph]

[BSW19a] Benini, M., Schenkel, A., Woike, L.: Involutive categories, colored $*$-operads and quantum field theory. Theor. Appl. Categ. 34, 13 (2019). arXiv:1802.09555 [math.CT]

[BSW19b] Benini, M., Schenkel, A., Woike, L.: Homotopy theory of algebraic quantum field theories. Lett. Math. Phys. 109(7), 1487 (2019). arXiv:1805.08795 [math-ph]

[BS18] Bruinsma, S., Schenkel, A.: Algebraic field theory operads and linear quantization. Lett. Math. Phys. 109(11), 2531 (2019). arXiv:1809.05319 [math-ph]

[BFV03] Brunetti, R., Fredenhagen, K., Verch, R.: The generally covariant locality principle: a new paradigm for local quantum field theory. Commun. Math. Phys. 237, 31 (2003). [math$\mathrm{ph} / 0112041]$

[CPTVV17] Calaque, D., Pantev, T., Toën, B., Vaquié, M., Vezzosi, G.: Shifted Poisson structures and deformation quantization. J. Topol. 10(2), 483 (2017). arXiv:1506.03699 [math.AG]

[CG17] Costello, K., Gwilliam, O.: Factorization Algebras in Quantum Field Theory. New Mathematical Monographs, vol. 31. Cambridge University Press, Cambridge (2017)

[DL12] Dappiaggi, C., Lang, B.: Quantization of Maxwell's equations on curved backgrounds and general local covariance. Lett. Math. Phys. 101, 265 (2012). arXiv:1104.1374 [gr-qc]

[DHKS04] Dwyer, W.G., Hirschhorn, P.S., Kan, D.M., Smith, J.H.: Homotopy Limit Functors on Model Categories and Homotopical Categories. Mathematical Surveys and Monographs, vol. 113. Amer. Math. Soc, Providence (2004)

[FH13] Fewster, C.J., Hunt, D.S.: Quantization of linearized gravity in cosmological vacuum spacetimes. Rev. Math. Phys. 25, 1330003 (2013). arXiv:1203.0261 [math-ph]

[FL16] Fewster, C.J., Lang, B.: Dynamical locality of the free Maxwell field. Annales Henri Poincaré 17(2), 401 (2016). arXiv:1403.7083 [math-ph]

[FR12] Fredenhagen, K., Rejzner, K.: Batalin-Vilkovisky formalism in the functional approach to classical field theory. Commun. Math. Phys. 314, 93 (2012). arXiv:1101.5112 [math-ph]

[FR13] Fredenhagen, K., Rejzner, K.: Batalin-Vilkovisky formalism in perturbative algebraic quantum field theory. Commun. Math. Phys. 317, 697 (2013). arXiv:1110.5232 [math-ph]

[HK64] Haag, R., Kastler, D.: An algebraic approach to quantum field theory. J. Math. Phys. 5, 848 (1964)

[HS13] Hack, T.P., Schenkel, A.: Linear bosonic and fermionic quantum gauge theories on curved spacetimes. Gen. Rel. Grav. 45, 877 (2013). arXiv:1205.3484 [math-ph]

[Hir03] Hirschhorn, P.S.: Model Categories and Their Localizations. Mathematical Surveys and Monographs, vol. 99. Amer. Math. Soc, Providence (2003)

[Hol08] Hollands, S.: Renormalized quantum Yang-Mills fields in curved spacetime. Rev. Math. Phys. 20, 1033 (2008). arXiv:0705.3340 [gr-qc]

[Hov99] Hovey, M.: Model Categories. Mathematical Surveys and Monographs, vol. 63. Amer. Math. Soc, Providence (1999)

[Kha16] Khavkine, I.: Cohomology with causally restricted supports. Annales Henri Poincaré 17(12), 3577 (2016). arXiv:1404.1932 [math-ph] 
[Kha18] Khavkine, I.: Compatibility complexes of overdetermined PDEs of finite type, with applications to the Killing equation. Class. Quant. Grav. 36, 185012 (2019). arXiv:1805.03751 [gr-qc]

[LurHTT] Lurie, J.: Higher Topos Theory. Annals of Mathematics Studies, vol. 170. Princeton University Press, Princeton (2009)

[LurHA] Lurie, J.: Higher algebra. http://www.math.harvard.edu/ lurie/papers/HA.pdf (2017)

[PTVV13] Pantev, T., Toën, B., Vaquié, M., Vezzosi, G.: Shifted symplectic structures. Publ. Math. Inst. Hautes Études Sci. 117, 271 (2013). arXiv:1111.3209 [math.AG]

[Pri18] Pridham, J.: An outline of shifted Poisson structures and deformation quantisation in derived differential geometry (2018). arXiv:1804.07622 [math.DG]

[Rie14] Riehl, E.: Categorical Homotopy Theory. New Mathematical Monographs, vol. 24. Cambridge University Press, Cambridge (2014)

[SDH14] Sanders, K., Dappiaggi, C., Hack, T.P.: Electromagnetism, local covariance, the AharonovBohm effect and Gauss' law. Commun. Math. Phys. 328, 625 (2014). arXiv:1211.6420 [math$\mathrm{ph}]$

[Sch13] Schreiber, U.: Differential cohomology in a cohesive infinity-topos (2013). https://ncatlab.org/ schreiber/show/differential+cohomology+in+a+cohesive+topos. arXiv:1310.7930 [math-ph]

[TZ18] Tehrani, M.T., Zahn, J.: Background independence in gauge theories (2018). arXiv:1804.07640 [math-ph]

[Wei94] Weibel, C.A.: An Introduction to Homological Algebra. Cambridge Studies in Advanced Mathematics, vol. 38. Cambridge University Press, Cambridge (1994)

Communicated by C. Schweigert 